\title{
ON THE TRANSITION DENSITIES FOR REFLECTED DIFFUSIONS
}

\author{
VADIM LINETSKY,$^{*}$ Northwestern University
}

\begin{abstract}
Diffusion models in economics, finance, queueing, mathematical biology, and electrical engineering often involve reflecting barriers. In this paper, we study the analytical representation of transition densities for reflected one-dimensional diffusions in terms of their associated Sturm-Liouville spectral expansions. In particular, we provide explicit analytical expressions for transition densities of Brownian motion with drift, the OrnsteinUhlenbeck process, and affine (square-root) diffusion with one or two reflecting barriers. The results are easily implementable on a personal computer and should prove useful in applications.
\end{abstract}

Keywords: Reflected diffusion; reflected Brownian motion; reflected Ornstein-Uhlenbeck process; reflected affine process; spectral expansion; currency target zone; heavy traffic

2000 Mathematics Subject Classification: Primary 60J35; 60J60; 60J70; 60K25; 91B28

\section{Introduction}

Diffusions with one or two reflecting barriers appear in many applications in economics, finance, queueing, mathematical biology, and electrical engineering. Among economics and finance applications, we mention the currency exchange rate target-zone models pioneered by Krugman (1991) (see also Svensson (1991), Bertolla and Caballero (1992), de Jong (1994), and Ball and Roma (1998)), in which the currency exchange rate is allowed to float within a target zone with two barriers enforced by the monetary authority; asset pricing models with price caps and/or price supports (e.g. price supports for agricultural commodities (see Hanson et al. (1999)); interest rate models with targeting by the monetary authority (e.g. Farnsworth and Bass (2003)); interest rate models with reflection at zero interest rate (e.g. Goldstein and Keirstead (1997) and Gorovoi and Linetsky (2004)); and stochastic volatility models (e.g. Schobel and Zhu (1999)). References to further applications in economics can be found in Veestraeten (2004).

In queueing theory, diffusions with reflecting barriers arise as heavy-traffic approximations of queueing systems. Reflected Brownian motion has long played a key role in queueing models (Harrison (1985), Abate and Whitt (1987a), (1987b)). More recently, reflected OrnsteinUhlenbeck (OU) and reflected affine processes have been studied as approximations of queueing systems with reneging or balking (Ward and Glynn (2003a), (2003b)) and multiserver loss

Received 29 June 2004; revision received 7 December 2004.

* Postal address: Department of Industrial Engineering and Management Sciences, McCormick School of Engineering and Applied Sciences, Northwestern University, 2145 Sheridan Road, Evanston, IL 60208, USA.

Email address: linetsky@iems.northwestern.edu 
models (Srikant and Whitt (1996)). Applications of reflected Ornstein-Uhlenbeck processes in mathematical biology were discussed in Ricciardi and Sacerdote (1987).

Explicit knowledge of the transition density of a process is important for the study of its transient properties and for statistical estimation of the process parameters from empirical data. The present paper studies the analytical representation of transition densities for reflected one-dimensional diffusions in terms of their associated Sturm-Liouville spectral expansions.

Applications of spectral theory to diffusions go back to McKean (1956) (see also Itô and McKean (1974, Section 4.11) and Wong (1964)). Based on the work of Feller on onedimensional diffusions, McKean constructed a spectral representation for a general one-dimensional diffusion. At around the same time, Karlin and McGregor (1957) constructed spectral representations for birth-and-death processes. The spectral expansion provides an analytical representation of the transition density. Roughly speaking, the spectral expansion may be interpreted as a large-time expansion in which the first term, corresponding to the zero principal eigenvalue, gives the steady-state density (if it exists), while the terms corresponding to the higher eigenvalues provide finite-time corrections to the steady-state density. Thus, we are able to study how the process converges to the steady state. Moreover, the explicit analytical knowledge of the transition density facilitates the maximum likelihood estimation of the process parameters from empirical data. A range of recent applications of spectral expansions to diffusion models in finance can be found in Lewis (1998), Davydov and Linetsky (2003), Gorovoi and Linetsky (2004), Linetsky (2004a), (2004b), (2004c), (2004d), (2004e), and references therein.

The paper is organized as follows. In Section 2, we give spectral representation results for one-dimensional diffusions with general drift and diffusion parameters and reflection at the origin. In Section 3, we give the large- $n$ asymptotics for eigenvalues and eigenfunctions for diffusions on a finite interval, reflected at both ends. In Section 4, we illustrate our results with examples of Brownian motion with drift on a finite interval, reflected at both ends, and on $[0, \infty)$, reflected at the origin. In Section 5, we provide an analytical treatment of the OrnsteinUhlenbeck process on the finite interval, reflected at both ends, and on $[0, \infty)$, reflected at the origin. In Section 6, we provide an analytical treatment of affine (square-root) diffusions on the finite interval, reflected at both ends, and on $[0, \infty)$, reflected at the origin. These results should be particularly useful for economics, finance, queueing, and mathematical biology, as OU and affine diffusions are widely used in these areas. In Section 7, we illustrate our results with numerical examples of reflected OU and affine diffusions computed in MATHEMATICA ${ }^{\circledR}$, and show that spectral expansions provide a powerful computational tool to study the transient behaviour of reflected diffusions. The Bessel processes with drift that appeared in queueing applications in Coffman et al. (1998) are treated in a companion paper Linetsky (2004e).

\section{Spectral representation of reflected diffusions}

Consider a one-dimensional regular, time-homogeneous diffusion $\left\{X_{t}, t \geq 0\right\}$ with state space $I=[0, r]$ or $I=[0, r), r \leq \infty$, and infinitesimal generator

$$
(g f)(x)=\frac{1}{2} a^{2}(x) f^{\prime \prime}(x)+b(x) f^{\prime}(x), \quad x \in(0, r),
$$

acting on functions on $I$ subject to appropriate regularity and boundary conditions.

Assumption 1. We assume that the diffusion coefficient $a(x)$ is continuous and strictly positive on $[0, r)$ and that the drift coefficient $b(x)$ is continuous on $[0, r)$. 
The infinitesimal generator can be rewritten as

$$
(g f)(x)=\frac{1}{\mathfrak{m}(x)}\left(\frac{f^{\prime}(x)}{\mathfrak{s}(x)}\right)^{\prime}, \quad x \in(0, r),
$$

where $\mathfrak{s}(x)$ and $\mathfrak{m}(x)$ are the scale and speed densities (see Borodin and Salminen (1996) or Karlin and Taylor (1981) for details):

$$
\mathfrak{s}(x)=\exp \left(-\int^{x} \frac{2 b(y)}{a^{2}(y)} \mathrm{d} y\right), \quad \mathfrak{m}(x)=\frac{2}{a^{2}(x) \mathfrak{s}(x)} .
$$

The densities $\mathfrak{s}(x)$ and $\mathfrak{m}(x)$ are defined up to a scaling constant, i.e. $\mathfrak{s}(x) \rightarrow c \mathfrak{s}(x)$ and $\mathfrak{m}(x) \rightarrow c^{-1} \mathfrak{m}(x)$.

Assumption 2. We assume that the origin is a regular instantaneously reflecting boundary included in the state space and that $(g f)(0):=\lim _{x \downarrow 0}(g f)(x)$. The right-hand boundary $r \leq \infty$ is either regular instantaneously reflecting, in which case it is included in the state space $I=[0, r]$ and $(g f)(r):=\lim _{x \uparrow r}(g f)(x)$, entrance, or nonattracting natural with

$$
\int_{0}^{r} \mathfrak{m}(x) \mathrm{d} x<\infty .
$$

If $r$ is entrance or natural, it is not included in the state space $I=[0, r)$.

Under these assumptions, $X$ has a stationary distribution with density

$$
\pi(x)=c^{-1} \mathfrak{m}(x), \quad c:=\int_{0}^{r} \mathfrak{m}(x) \mathrm{d} x .
$$

We can choose the scaling constant in the definition of $\mathfrak{s}(x)$ and $\mathfrak{m}(x)$ so that $\int_{0}^{r} \mathfrak{m}(x) \mathrm{d} x=1$ but, for future convenience, we will not do so.

Let $C_{\mathrm{b}}(I)$ be the Banach space of real-valued, bounded continuous functions on $I$. Then, the conditional expectation operators

$$
\left(\mathcal{P}_{t} f\right)(x):=\mathrm{E}_{x}\left[f\left(X_{t}\right)\right]=\int_{I} f(y) p(t ; x, y) \mathrm{d} y
$$

(where $\mathrm{E}_{x}[\cdot] \equiv \mathrm{E}\left[\cdot \mid X_{0}=x\right]$ ) form a Feller semigroup $\left\{\mathcal{P}_{t}, t \geq 0\right\}$ in $C_{\mathrm{b}}(I)$. Here, $p(t ; x, y)$ is the transition density with respect to the Lebesgue measure. The domain of the infinitesimal generator $g$ of $\left\{\mathcal{P}_{t}, t \geq 0\right\}$ in $C_{\mathrm{b}}(I)$ is

$$
D(g):=\left\{f \in C_{\mathrm{b}}(I): g f \in C_{\mathrm{b}}(I), \text { boundary conditions at } 0 \text { and } r\right\} .
$$

The boundary condition at 0 is

$$
f^{\prime}(0)=0 .
$$

If $r$ is regular instantaneously reflecting or entrance, then the boundary condition can be written in the form

$$
\lim _{x \uparrow r} \frac{f^{\prime}(x)}{\mathfrak{s}(x)}=0 .
$$

If $r$ is natural, then no boundary condition is needed there. 
Let $L^{2}(I, \mathfrak{m})$ be the Hilbert space of real-valued functions on $I$ square integrable on $I$ with weight $\mathfrak{m}$ and with inner product

$$
(f, g)=\int_{I} f(x) g(x) \mathfrak{m}(x) \mathrm{d} x .
$$

The Feller semigroup $\left\{\mathcal{P}_{t}, t \geq 0\right\}$ restricted to $C_{\mathrm{b}}(I) \cap L^{2}(I, \mathfrak{m})$ extends uniquely to a selfadjoint contraction semigroup on $L^{2}(I, \mathfrak{m})$ with the infinitesimal generator $g$, an unbounded self-adjoint, nonpositive operator in $L^{2}(I, \mathfrak{m})$ (McKean (1956); see also Itô and McKean (1974, Section 4.11) and Langer and Schenk (1990)). The spectral decomposition of $g$ in $L^{2}(I, \mathfrak{m})$ yields the spectral decomposition of the semigroup $\mathcal{P}_{t}$ and the spectral representation of the transition density $p(t ; x, y)$ :

$$
p(t ; x, y)=\mathfrak{m}(x) \int_{[0, \infty)} \mathrm{e}^{-\lambda t} \psi(x, \lambda) \psi(y, \lambda) \mathrm{d} \rho(\lambda), \quad t>0 .
$$

In the spectral expansion (4), $\psi(x, \lambda)$ is the unique solution of the Sturm-Liouville (SL) equation

$$
-(g u)(x)=\lambda u(x)
$$

with the initial conditions

$$
u(0)=1, \quad u^{\prime}(0)=0,
$$

and $\rho(\lambda)$ is the (nondecreasing, right-continuous) spectral function of the SL operator $\mathcal{A}=-\mathcal{G}$ (i.e. the negative of the infinitesimal generator $g$ ). The integral in (4) converges uniformly in $x$ and $y$ on compact squares in $I \times I$ (McKean (1956)).

The problem is thus reduced to determining the spectral function $\rho(\lambda)$ and the solution $\psi(x, \lambda)$ of the initial value problem. When $r$ is not a natural boundary, the spectrum of $g$ is purely discrete (McKean (1956, Theorem 3.1)). When $r$ is a natural boundary, the situation is more complicated and there may be a nonempty continuous region in the spectrum. The foregoing discussion of natural boundaries follows Linetsky (2004d). Natural boundaries can be classified into two further subcategories, based on the oscillation of solutions of the associated SL equation (5). For a given real $\lambda,(5)$ is said to be oscillatory at an endpoint $e$ if and only if every solution has infinitely many zeros clustered at $e$. Otherwise, it is said to be nonoscillatory at $e$. These subcategories are mutually exclusive for a fixed real $\lambda$, but the classification can vary with $\lambda$. Generally, if an endpoint is a regular, exit, or entrance boundary for the diffusion process, then the associated SL equation is nonoscillatory for all real $\lambda$ at that endpoint. In our case, 0 is regular reflecting and, hence, the SL equation is nonoscillatory at 0 for all $\lambda$. If $r$ is regular reflecting or entrance, then the SL equation is nonoscillatory at $r$ for all $\lambda$. If $r$ is natural, there are two alternatives: either (i) (5) is nonoscillatory at $r$ for all real $\lambda$ (correspondingly, $r$ is called nonoscillatory) or (ii) there exists a real number $\Lambda \geq 0$ such that (5) is oscillatory at $r$ for all $\lambda>\Lambda$ and nonoscillatory at $r$ for all $\lambda<\Lambda$ (correspondingly, $r$ is called oscillatory with cutoff $\Lambda$ ). Equation (5) can be either oscillatory or nonoscillatory at $r$ for $\lambda=\Lambda>0$. It is always nonoscillatory for $\lambda=0$.

Based on this classification of boundaries, the spectrum of the SL operator $\mathcal{A}$ associated with the diffusion process $X$ on $[0, r)$, with reflecting boundary at 0 and natural boundary at $r$, is classified as follows: (i) if $r$ is nonoscillatory then the spectrum is simple, nonnegative, and purely discrete, while (ii) if $r$ is oscillatory, with cutoff $\Lambda \geq 0$, then the spectrum is simple and nonnegative, the essential spectrum is nonempty, $\sigma_{e}(\mathcal{A}) \subseteq[\Lambda, \infty)$, and $\Lambda$ is the lowest 
point of the essential spectrum. (A real value $\lambda \in \mathbb{R}$ is said to be in the essential spectrum of a self-adjoint differential operator if and only if one or both of the following hold: (a) $\lambda$ is in the continuous spectrum; (b) $\lambda$ is a limit point of the point spectrum.) Furthermore, if the SL equation is nonoscillatory at $r$ for $\lambda=\Lambda \geq 0$, then there is a finite set of simple eigenvalues in $[0, \Lambda]$ (under Assumption 2, the set contains at least the principal eigenvalue $\lambda_{0}=0$ ). If the SL equation is oscillatory at $r$ for $\lambda=\Lambda>0$, then there is an infinite sequence of simple eigenvalues in $[0, \Lambda)$, with the limit point $\Lambda$.

Thus, nonoscillatory natural boundaries are similar to regular, exit, and entrance boundaries in that they do not generate any continuous spectrum. Suppose that Assumptions 1 and 2 are satisfied and that $r$ is either a regular instantaneously reflecting, entrance, or nonoscillatory nonattracting natural boundary. Let $\lambda_{0}=0<\lambda_{1}<\lambda_{2}<\cdots$ be the eigenvalues of the SL operator $\mathcal{A}$. (Note that the principal eigenvalue is $\lambda_{0}=0$ since constants satisfy the SL equation (5) with $\lambda=0$ and the boundary conditions at 0 and $r$.) Then the spectral function can be written in the form

$$
\rho(\lambda)=\sum_{n=0}^{\infty} \frac{1}{\left\|\psi\left(\cdot, \lambda_{n}\right)\right\|^{2}} \mathbf{1}_{\left\{\lambda_{n} \leq \lambda\right\}}
$$

where $\mathbf{1}_{\left\{\lambda_{n} \leq \lambda\right\}}=1$ if $\lambda_{n} \leq \lambda$ and $\mathbf{1}_{\left\{\lambda_{n} \leq \lambda\right\}}=0$ if $\lambda_{n}>\lambda$, and $\|\cdot\|$ is the $L^{2}(I, \mathfrak{m})$ norm. It jumps by $\left\|\psi\left(\cdot, \lambda_{n}\right)\right\|^{-2}$ at an eigenvalue $\lambda=\lambda_{n}$. The spectral representation (4) thus reduces to the eigenfunction expansion

$$
p(t ; x, y)=\pi(y)+\mathfrak{m}(y) \sum_{n=1}^{\infty} \mathrm{e}^{-\lambda_{n} t} \varphi_{n}(x) \varphi_{n}(y), \quad t>0,
$$

where the sum converges uniformly in $x$ and $y$ on compact squares in $I \times I$. Here, $\varphi_{n}$ are the normalized eigenfunctions given by (up to an overall sign) $\varphi_{n}(x)= \pm \psi\left(x, \lambda_{n}\right) /\left\|\psi\left(\cdot, \lambda_{n}\right)\right\|$. The first term in the expansion, corresponding to the principal eigenvalue $\lambda_{0}=0$, is the stationary density (1) (note that $\psi(x, 0)=1$ ).

Thus, the problem is reduced to determining the eigenvalues $\lambda_{n}$ and eigenfunctions $\varphi_{n}$.

Proposition 1. For $\lambda \in \mathbb{C}$ and $x \in I$, let $\phi(x, \lambda)$ be the unique (up to a factor independent of $x$ ) nontrivial solution of the SL equation (5) square integrable with weight $\mathfrak{m}$ near $r$, satisfying the boundary condition (3) at $r$, and such that $\phi(x, \lambda)$ and $\phi^{\prime}(x, \lambda) \equiv \partial \phi(x, \lambda) / \partial x$ are continuous in $x$ and $\lambda$ in $I \times \mathbb{C}$ and entire in $\lambda$ for each fixed $x \in I$. Then the eigenvalues $\left\{\lambda_{n}, n=0,1, \ldots\right\}$ can be identified with simple nonnegative zeros of $\phi^{\prime}(0, \lambda)$, i.e. roots of the equation

$$
\phi^{\prime}(0, \lambda)=0
$$

and the normalized eigenfunctions can be written in the form

$$
\varphi_{n}(x)= \pm \sqrt{\frac{\mathfrak{s}(0)}{A_{n} \delta_{n}}} \phi\left(x, \lambda_{n}\right)= \pm \sqrt{\frac{\mathfrak{s}(0) A_{n}}{\delta_{n}}} \psi\left(x, \lambda_{n}\right),
$$

where

$$
A_{n}:=\phi\left(0, \lambda_{n}\right), \quad \delta_{n}:=\left.\frac{\mathrm{d} \phi^{\prime}(0, \lambda)}{\mathrm{d} \lambda}\right|_{\lambda=\lambda_{n}} .
$$


Proof. The solution $\phi(x, \lambda)$ with the required properties exists by Lemma 1 in Linetsky (2004d). For $\lambda \in \mathbb{C}$ and $x \in I$, let $\psi(x, \lambda)$ be the unique solution of the SL equation (5) with the initial conditions (6). Both $\psi(x, \lambda)$ and $\psi^{\prime}(x, \lambda)$ are continuous in $x$ and $\lambda$ in $I \times \mathbb{C}$ and entire in $\lambda$ for each fixed $x \in I$. Since $\psi(x, \lambda)$ and $\phi(x, \lambda)$ are solutions of the SL equation, and $\psi(x, \lambda)$ satisfies the initial conditions $(6)$, the Wronskian of $\psi(x, \lambda)$ and $\phi(x, \lambda)$ is independent of $x$ and is entire in $\lambda$ :

$$
\psi(x, \lambda) \frac{\phi^{\prime}(x, \lambda)}{\mathfrak{s}(x)}-\phi(x, \lambda) \frac{\psi^{\prime}(x, \lambda)}{\mathfrak{s}(x)}=: w(\lambda) .
$$

Setting $x=0$ and using the initial condition (6), we have for the Wronskian

$$
w(\lambda)=\frac{\phi^{\prime}(0, \lambda)}{\mathfrak{s}(0)} .
$$

The eigenfunction $\varphi_{n}$ satisfies the boundary condition (2) at 0 ; hence, it must be equal to $\psi\left(x, \lambda_{n}\right)$ up to a nonzero constant multiple. However, $\varphi_{n}(x)$ also satisfies the boundary condition at $r$ and, hence, it must also be equal to $\phi\left(x, \lambda_{n}\right)$ up to a nonzero constant multiple. Thus, for $\lambda=\lambda_{n}, \psi\left(x, \lambda_{n}\right)$ and $\phi\left(x, \lambda_{n}\right)$ are linearly dependent, $\phi\left(x, \lambda_{n}\right)=A_{n} \psi\left(x, \lambda_{n}\right)$, and, hence, their Wronskian must vanish for $\lambda=\lambda_{n}$. Setting $x=0$ and using the initial condition at 0 , we find that $A_{n}=\phi\left(0, \lambda_{n}\right)$. Thus, from (11), the eigenvalues can be identified with the zeros of $\phi^{\prime}(0, \lambda)$. Conversely, let $\lambda_{n}$ be a zero of $\phi^{\prime}(0, \lambda)$. Then $\psi\left(x, \lambda_{n}\right)$ and $\phi\left(x, \lambda_{n}\right)$ are linearly dependent and, hence, $\psi\left(x, \lambda_{n}\right)$ is a solution of the SL equation that is square integrable with weight $\mathfrak{m}$ on $I$ and satisfies the required boundary conditions at 0 and $r$, i.e. $\psi\left(x, \lambda_{n}\right)$ is a (nonnormalized) eigenfunction corresponding to the eigenvalue $\lambda_{n}$. Finally, up to an overall sign, the normalized eigenfunctions can be written in the form

$$
\varphi_{n}(x)= \pm \frac{\psi\left(x, \lambda_{n}\right)}{\left\|\psi\left(\cdot, \lambda_{n}\right)\right\|}= \pm \frac{\phi\left(x, \lambda_{n}\right)}{\left\|\phi\left(\cdot, \lambda_{n}\right)\right\|},
$$

where the norms can be calculated analytically (see Lemma 2 in Linetsky (2004d), with $\left.\left.w^{\prime}\left(\lambda_{n}\right) \equiv(\mathrm{d} w(\lambda) / \mathrm{d} \lambda)\right|_{\lambda=\lambda_{n}}\right)$, giving

$$
\left\|\psi\left(\cdot, \lambda_{n}\right)\right\|^{2}=\frac{w^{\prime}\left(\lambda_{n}\right)}{A_{n}}=\frac{\delta_{n}}{\mathfrak{s}(0) A_{n}}, \quad\left\|\phi\left(\cdot, \lambda_{n}\right)\right\|^{2}=w^{\prime}\left(\lambda_{n}\right) A_{n}=\frac{A_{n} \delta_{n}}{\mathfrak{s}(0)},
$$

where $\delta_{n}$ and $A_{n}$ are as defined in (10).

Thus, when $r$ is nonoscillatory, the problem is reduced to determining the zeros $\lambda_{n}$ of $\phi^{\prime}(0, \lambda)$. We now study the case in which $r$ is an oscillatory natural boundary with cutoff $\Lambda \geq 0$. Sufficient conditions for the oscillatory/nonoscillatory classification of natural boundaries can be formulated directly in terms of the behaviour of $a(x)$ and $b(x)$ near the boundary $r$ (Linetsky (2004d)). We first transform the SL equation (5) to the Liouville normal form. The following smoothness assumptions are sufficient to be able to perform the Liouville transformation.

Assumption 3. The functions $a^{\prime}(x), a^{\prime \prime}(x)$, and $b^{\prime}(x)$ exist and are continuous on $[0, r)$.

We transform the independent and dependent variables as follows, where $x=x(y)$ is the inverse of the Liouville transformation $y=y(x)$ :

$$
y=y(x)=\sqrt{2} \int_{0}^{x} \frac{\mathrm{d} z}{a(z)}, \quad v(y)=\frac{2^{1 / 4} u(x(y))}{\sqrt{a(x(y)) \mathfrak{s}(x(y))}} .
$$


The function $v(y)$ then satisfies the SL equation in the Liouville normal form (in which the coefficient in front of the second derivative is constant and equal to negative one and the firstderivative term is absent; the SL equation in the Liouville normal form has the form of the one-dimensional Schrödinger equation):

$$
-v^{\prime \prime}+Q(y) v=\lambda v, \quad y \in(0, R), \quad R:=y(r) .
$$

Here, the potential function $Q(y)$ is given by

$$
Q(y)=\mathcal{V}(x(y))
$$

where

$$
\mathcal{V}(x):=\frac{1}{8}\left(a^{\prime}(x)\right)^{2}-\frac{1}{4} a(x) a^{\prime \prime}(x)+\frac{b^{2}(x)}{2 a^{2}(x)}+\frac{1}{2} b^{\prime}(x)-\frac{b(x) a^{\prime}(x)}{a(x)},
$$

and is continuous on $[0, r)$.

The oscillatory/nonoscillatory classification remains invariant under the Liouville transformation, i.e. (5) is nonoscillatory at $r$ for a particular $\lambda$ if and only if (5) is nonoscillatory at $R$ for that $\lambda$. The oscillatory/nonoscillatory classification of (5) depends on the behaviour of the potential function $Q$ near $R$. To formulate the classification result, we make the following additional assumption.

Assumption 4. Let $r$ be a natural boundary. We assume that the limit $\lim _{x \uparrow r} \mathcal{V}(x)$ exists (it is allowed to be infinite).

Under this assumption, we have the following two alternatives. (i) Suppose that $r$ is transformed into a finite endpoint by the Liouville transformation, i.e. $R<\infty$; then $r$ is nonoscillatory. (ii) Suppose that $r$ is transformed into $R=\infty$ by the Liouville transformation. If

$$
\lim _{x \uparrow r} \mathcal{V}(x)=\infty
$$

then $r$ is nonoscillatory. If, for some finite $\Lambda$,

$$
\lim _{x \uparrow r} \mathcal{V}(x)=\Lambda,
$$

then $r$ is oscillatory with cutoff $\Lambda$. Since the SL operator $\mathcal{A}$ is nonnegative, it follows that $\Lambda \geq 0$. If $\Lambda=0, r$ is always nonoscillatory for $\lambda=\Lambda=0$. To determine whether $r$ is oscillatory or nonoscillatory for $\lambda=\Lambda>0$, we have the following criterion. If

$$
\lim _{y \uparrow R} y^{2}(Q(y)-\Lambda)>-\frac{1}{4},
$$

then $r$ is nonoscillatory for $\lambda=\Lambda>0$, while if

$$
\lim _{y \uparrow R} y^{2}(Q(y)-\Lambda)<-\frac{1}{4},
$$

then $r$ is oscillatory for $\lambda=\Lambda>0$.

When $r$ is an oscillatory natural boundary with cutoff $\Lambda \geq 0$, there is some nonempty essential spectrum above $\Lambda$. In general, the essential spectrum may have a complicated structure. In particular, if the potential function oscillates towards an infinite boundary, it may consist of an infinite sequence of disjoint intervals separated by gaps. Furthermore, eigenvalues may be present in the gaps or embedded in the continuous spectrum. The following assumption simplifies the structure of the essential spectrum. 
Assumption 5. If $r$ is an oscillatory natural boundary with cutoff $\Lambda$, we assume that the function $\mathcal{V}(x)$ has bounded variation on $[0, r)$.

With this assumption, the essential spectrum of $\mathcal{A}$ is $\sigma_{e}(\mathcal{A})=[\Lambda, \infty)$. Moreover, the spectrum above $\Lambda$ is purely absolutely continuous (see Linetsky (2004d)), and the spectral function $\rho(\lambda)$ can be written in the form

$$
\rho(\lambda)=\sum_{n \geq 0} \frac{1}{\left\|\psi\left(\cdot, \lambda_{n}\right)\right\|^{2}} \mathbf{1}_{\left\{\lambda_{n} \leq \lambda\right\}}+\rho_{\mathrm{ac}}(\lambda) \mathbf{1}_{\{\Lambda \leq \lambda\}}
$$

Here, $\rho_{\mathrm{ac}}(\lambda)$ is the absolutely continuous part and $\left\{\lambda_{n}\right\}$ are the eigenvalues in $[0, \Lambda]$. If $r$ is nonoscillatory for $\lambda=\Lambda$ then this set is finite and contains at least the principal eigenvalue $\lambda_{0}=0$. If $r$ is oscillatory for $\lambda=\Lambda$ then the set is infinite; there is an infinite sequence of eigenvalues starting with $\lambda_{0}=0$ and increasing towards $\Lambda$ - the limit point of this sequence. In both cases, the spectral expansion for the density reduces to

$$
p(t ; x, y)=\pi(y)+\mathfrak{m}(y) \sum_{n \geq 1} \mathrm{e}^{-\lambda_{n} t} \varphi_{n}(x) \varphi_{n}(x)+\mathfrak{m}(y) \int_{\Lambda}^{\infty} \mathrm{e}^{-\lambda t} \psi(x, \lambda) \psi(y, \lambda) \mathrm{d} \rho_{\mathrm{ac}}(\lambda) .
$$

Remark 1. The spectral function (17) can be obtained as follows. Pick some $\varepsilon \in(0, r)$ and consider a diffusion process $X^{(\varepsilon)}$ reflected at $\varepsilon$. The right-hand endpoint $\varepsilon$ is regular reflecting and the spectral function $\rho^{(\varepsilon)}(\lambda)$ has the form (7). Then take the limit as $\varepsilon \rightarrow r$. The limit $\lim _{\varepsilon \uparrow r} \rho^{(\varepsilon)}(\lambda)=\rho(\lambda)$ produces the spectral function of the original problem on $[0, r)$ with natural boundary $r$. This is the standard approach of approximating a singular SL problem with a regular problem by placing a regular boundary just before the singular boundary (Levinson (1951), Levitan (1950), McKean (1956), Levitan and Sargsjan (1975)). The regularized problem has a purely discrete spectrum. The singular problem is recovered in the limit of taking the regular boundary towards the singular one. Roughly speaking, the discrete spectrum above $\Lambda$ of the regular problem on $[0, \varepsilon]$ merges into the continuous spectrum of the singular problem on $[0, r)$ in the limit as $\varepsilon \uparrow r$. (As $\varepsilon$ increases towards $r$, the eigenvalues are distributed more and more densely and, in the limit, merge to form the continuous spectrum.)

Remark 2. Equations (8) and (18) give the spectral representation of the transition density. It is straightforward to show that the cumulative distribution function has the spectral representation

$$
\mathrm{P}_{x}\left(X_{t} \leq y\right)=\mathrm{P}^{\pi}\left(X_{\infty} \leq y\right)-\sum_{n=1}^{\infty} \mathrm{e}^{-\lambda_{n} t} \frac{\varphi_{n}(x) \varphi_{n}^{\prime}(y)}{\lambda_{n} \mathfrak{s}(y)}, \quad t>0, x \in I,
$$

when $r$ is nonoscillatory, and

$$
\mathrm{P}_{x}\left(X_{t} \leq y\right)=\mathrm{P}^{\pi}\left(X_{\infty} \leq y\right)-\sum_{n \geq 1} \mathrm{e}^{-\lambda_{n} t} \frac{\varphi_{n}(x) \varphi_{n}^{\prime}(y)}{\lambda_{n} \mathfrak{s}(y)}-\int_{\Lambda}^{\infty} \mathrm{e}^{-\lambda t} \frac{\psi(x, \lambda) \psi^{\prime}(y, \lambda)}{\lambda \mathfrak{s}(y)} \mathrm{d} \rho_{\mathrm{ac}}(\lambda)
$$

when $r$ is oscillatory with cutoff $\Lambda \geq 0$. Here, $\mathrm{P}^{\pi}\left(X_{\infty} \leq y\right)=\int_{0}^{y} \pi(x) \mathrm{d} x$.

Remark 3. In this section we have made a number of assumptions on the drift and diffusion coefficients of the process and its boundary behaviour (Assumptions 1-5). Here we comment on each of the assumptions in turn. Continuity of $a(x)$ and $b(x)$ (Assumption 1) is not necessary 
in order to develop the general form of the spectral representation for transition density. In fact, McKean's (1956) spectral representation is valid in the general case of diffusion defined by scale and speed measures that are not required to be absolutely continuous with respect to the Lebesgue measure (e.g. Borodin and Salminen (1996, pp. 16-17)). Moreover, the spectral representation generalizes to gap diffusions (e.g. Langer and Schenk (1990)). We made the simplifying assumption of continuous coefficients in order to be able to apply standard results from Sturm-Liouville theory that require continuity of coefficients.

Furthermore, we restricted ourselves to processes with the right-hand boundary $r$ being reflecting, entrance, or nonattracting natural (Assumption 2). Under this assumption, the process has a stationary density. This is a case often encountered in applications of reflected diffusions. However, all results in this paper can be directly modified to extend to the cases in which $r$ is either killing (e.g. an OU process reflected at 0 and killed at $r<\infty$; see Linetsky (2004a) for details on the killed OU process) or attracting natural (e.g. Brownian motion on $[0, \infty)$, reflected at 0 , with positive drift). In the former case, the boundary condition (3) at $r$ changes to $f(r)=0$ (in the latter case, no boundary condition at $r$ is necessary). In these cases, the process does not have a stationary distribution and zero is no longer the principal eigenvalue (the lower bound of the spectrum is strictly positive). See Linetsky (2004d) for more details.

Assumption 3, of further continuity of $a(x)$ and $b(x)$, is only needed in order to be able to perform the Liouville transformation to the Schrödinger form (13), and Assumptions 4 and 5, of the existence of the limit and the bounded variation of $\mathcal{V}(x)$, are only needed in order to formulate a simple and explicit classification of the spectrum. These assumptions are satisfied in most applications (e.g. OU and affine processes). However, in general, these assumptions are not required in order to be able to construct the spectral representation. In fact, an example of a process with constant diffusion coefficient, drift $m(x)=-p \mu \beta$ for $0 \leq x<p \kappa$ and $m(x)=-p \mu(x+\beta)$ for $x<0$, discontinuous first derivative, and a reflecting boundary condition at $p \kappa$ appears in Whitt (2004, Theorem 2.1) and Whitt (2005, Theorem 2.1) in queueing applications ( $\mu$ and $\beta$ are constant parameters). In this case, we cannot reduce the Sturm-Liouville equation to the Schrödinger form (13) with continuous potential, but we can still either work with the original SL equation (5) directly, or with the Schrödinger equation with discontinuous potential, to construct the corresponding spectral expansion.

\section{Large- $n$ eigenvalue and eigenfunction asymptotics for diffusions reflected at both boundaries}

Consider a diffusion on $[0, r]$ with both 0 and $r$ regular instantaneously reflecting boundaries and suppose that Assumptions 1 and 2 hold on $[0, r]$. Large- $n$ eigenvalue and eigenfunction asymptotics for regular SL operators are available in the Sturm-Liouville literature. The following results follow from the results in Fulton and Pruess (1994).

Consider the SL equation (5) with the boundary conditions $u^{\prime}(0)=0$ and $u^{\prime}(r)=0$. The Liouville transformation (12) transforms (5) to the Liouville normal form (13). The boundary conditions are transformed into the boundary conditions

$$
v^{\prime}(0)-\gamma_{1} v(0)=0, \quad v^{\prime}(R)-\gamma_{2} v(R)=0,
$$

where the coefficients $\gamma_{1}$ and $\gamma_{2}$ are given by

$$
\gamma(x):=\frac{1}{\sqrt{2}}\left(\frac{b(x)}{a(x)}-\frac{a^{\prime}(x)}{2}\right), \quad \gamma_{1}:=\gamma(0), \quad \gamma_{2}:=\gamma(r) .
$$


Let $Q(y)$ be the potential function (14) (in this section we assume that $Q^{\prime}(y)$ exists on $[0, R]$ ). We introduce the notation

$$
\begin{array}{rlrl}
C_{1} & :=\int_{0}^{R} Q(y) \mathrm{d} y, & C_{2}:=\int_{0}^{R} Q^{2}(y) \mathrm{d} y, \\
A(x) & :=\int_{0}^{y(x)} Q(z) \mathrm{d} z, \quad x \in[0, r],
\end{array}
$$

where

$$
y(x)=2^{1 / 2} \int_{0}^{x} a(z)^{-1} \mathrm{~d} z .
$$

We have the following large- $n$ asymptotics for the eigenvalues and normalized eigenfunctions:

$$
\begin{aligned}
\lambda_{n}= & \frac{n^{2} \pi^{2}}{R^{2}}+a_{0}+\frac{a_{2}}{n^{2} \pi^{2}}+O\left(\frac{1}{n^{4}}\right) \\
a_{0}= & \frac{1}{R}\left(2 \gamma_{1}-2 \gamma_{2}+C_{1}\right) \\
a_{2}= & \frac{1}{4} R\left(C_{2}+Q^{\prime}(R)-Q^{\prime}(0)\right)+R\left(\gamma_{1} Q(0)-\gamma_{2} Q(R)\right)-\frac{2}{3} R\left(\gamma_{1}^{3}-\gamma_{2}^{3}\right) \\
& -\left(\gamma_{1}-\gamma_{2}+\frac{1}{2} C_{1}\right)^{2} \\
\varphi_{n}(x)= & \pm 2^{1 / 4} \sqrt{\frac{a(x) \mathfrak{s}(x)}{R}} \\
& \quad \times\left\{\cos \left(\frac{n \pi y(x)}{R}\right)+\frac{R}{n \pi}\left(\frac{1}{2} A(x)+\gamma_{1}-\frac{1}{2} a_{0} y(x)\right) \sin \left(\frac{n \pi y(x)}{R}\right)\right\}+O\left(\frac{1}{n^{2}}\right) .
\end{aligned}
$$

These estimates are useful in applications. The large- $n$ asymptotics of the eigenvalues facilitate the numerical work of finding accurate eigenvalues as zeros of the Wronskian $w(\lambda)$. We can use the estimates as a starting point of some numerical search procedure to find the accurate values of $\lambda_{n}$. Often, even for moderate values of $n$, the estimates approximate the exact eigenvalues and eigenfunctions sufficiently closely. Here we have given the large- $n$ eigenvalue estimates with an error of the order $1 / n^{4}$. Additional terms in the $1 / n$ expansions can be obtained by following the procedure in Fulton and Pruess (1994).

\section{Reflected Brownian motion with drift}

\subsection{Brownian motion with drift on $[0, r]$, reflected at 0 and $r$}

Consider Brownian motion with constant drift $\mu \in \mathbb{R}$ on $[0, r]$, reflected at both 0 and $r$. The scale, speed, and stationary densities are

$$
\mathfrak{s}(x)=\mathrm{e}^{-2 \mu x}, \quad \mathfrak{m}(x)=2 \mathrm{e}^{2 \mu x}, \quad \pi(x)=\frac{2 \mu \mathrm{e}^{2 \mu x}}{\mathrm{e}^{2 \mu r}-1},
$$

respectively. The associated SL equation has constant coefficients:

$$
\frac{1}{2} u^{\prime \prime}+\mu u^{\prime}+\lambda u=0 .
$$


For $\lambda \in \mathbb{C}$ and $x \in[0, r]$, the solutions $\phi(x, \lambda)$ and $\psi(x, \lambda)$, with the initial conditions $\phi(r, \lambda)=1$ and $\phi^{\prime}(r, \lambda)=0$, and $\psi(0, \lambda)=1$ and $\psi^{\prime}(0, \lambda)=0$, and their Wronskian are

$$
\begin{aligned}
\phi(x, \lambda) & =\mathrm{e}^{\mu(r-x)}\left\{\cosh (v(\lambda)(r-x))-\frac{\mu}{v(\lambda)} \sinh (v(\lambda)(r-x))\right\}, \\
\psi(x, \lambda) & =\mathrm{e}^{-\mu x}\left\{\cosh (v(\lambda) x)+\frac{\mu}{v(\lambda)} \sinh (v(\lambda) x)\right\}, \\
w(\lambda) & =\mathrm{e}^{\mu r} \frac{2 \lambda}{v(\lambda)} \sinh (v(\lambda) r), \quad \text { where } v(\lambda)=\sqrt{\mu^{2}-2 \lambda .}
\end{aligned}
$$

The Wronskian zeros/eigenvalues are

$$
\lambda_{0}=0, \quad \lambda_{n}=\frac{\mu^{2}}{2}+\frac{\pi^{2} n^{2}}{2 r^{2}}, \quad n=1,2, \ldots
$$

Substituting $\lambda_{n}$ into $\phi(x, \lambda)$ gives $\phi\left(x, \lambda_{0}\right)=1$,

$$
\phi\left(x, \lambda_{n}\right)=(-1)^{n} \mathrm{e}^{\mu(r-x)}\left\{\cos \left(\frac{x \pi n}{r}\right)+\frac{\mu r}{\pi n} \sin \left(\frac{x \pi n}{r}\right)\right\}, \quad n=1,2, \ldots,
$$

and

$$
A_{0}=1, \quad A_{n}=\phi\left(0, \lambda_{n}\right)=(-1)^{n} \mathrm{e}^{\mu r}, \quad n=1,2, \ldots
$$

The Wronskian derivative evaluated at $\lambda_{n}$ is

$$
\delta_{n}=w^{\prime}\left(\lambda_{n}\right)=(-1)^{n} \mathrm{e}^{\mu r} r\left(1+\frac{\mu^{2} r^{2}}{\pi^{2} n^{2}}\right) .
$$

The normalized eigenfunctions (9) can be written in the form

$$
\varphi_{n}(x)= \pm \frac{\mathrm{e}^{-\mu x}}{\sqrt{r\left(1+\left(\mu^{2} r^{2} / \pi^{2} n^{2}\right)\right)}}\left\{\cos \left(\frac{x \pi n}{r}\right)+\frac{\mu r}{\pi n} \sin \left(\frac{x \pi n}{r}\right)\right\} .
$$

Substituting this result into (8) and (19), we obtain the density and the cumulative distribution function as follows:

$$
\begin{aligned}
p(t ; x, y)= & \frac{2 \mu \mathrm{e}^{2 \mu x}}{\mathrm{e}^{2 \mu r}-1}+\frac{2}{r} \mathrm{e}^{\mu(y-x)-\mu^{2} t / 2} \\
& \times \sum_{n=1}^{\infty} \frac{\mathrm{e}^{-\left(\pi^{2} n^{2} / 2 r^{2}\right) t}}{\mu^{2}+\pi^{2} n^{2} / r^{2}}\left\{\frac{\pi n}{r} \cos \left(\frac{x \pi n}{r}\right)+\mu \sin \left(\frac{x \pi n}{r}\right)\right\} \\
& \times\left\{\frac{\pi n}{r} \cos \left(\frac{y \pi n}{r}\right)+\mu \sin \left(\frac{y \pi n}{r}\right)\right\}
\end{aligned}
$$

and

$$
\begin{aligned}
\mathrm{P}_{x}\left(X_{t} \leq y\right)= & \frac{\mathrm{e}^{2 \mu y}-1}{\mathrm{e}^{2 \mu r}-1}+\frac{2}{r} \mathrm{e}^{\mu(y-x)-\mu^{2} t / 2} \\
& \times \sum_{n=1}^{\infty} \frac{\mathrm{e}^{-\left(\pi^{2} n^{2} / 2 r^{2}\right) t}}{\mu^{2}+\pi^{2} n^{2} / r^{2}}\left\{\frac{\pi n}{r} \cos \left(\frac{x \pi n}{r}\right)+\mu \sin \left(\frac{x \pi n}{r}\right)\right\} \sin \left(\frac{y \pi n}{r}\right) .
\end{aligned}
$$


For $\mu=0,(25)$ and (26) reduce to the familiar expressions for driftless Brownian motion on $[0, r]$, reflected at 0 and $r$ (e.g. Borodin and Salminen (1996)).

The Liouville transformation (12), i.e. $y=2^{1 / 2} x$ and $v(y)=2^{1 / 4} \exp \left(2^{-1 / 2} \mu y\right) u\left(2^{-1 / 2} y\right)$, reduces (23) to the Schrödinger equation (with constant potential)

$$
v^{\prime \prime}+(\lambda-Q) v=0, \quad Q=\frac{1}{2} \mu^{2}, \quad x \in(0, R), R=\sqrt{2} r,
$$

subject to the boundary conditions

$$
v^{\prime}(0)-\gamma v(0)=0, \quad v^{\prime}(R)-\gamma v(R)=0, \quad \gamma=\mu / \sqrt{2} .
$$

In this case, the estimate (21) for the eigenvalues is exact with $a_{n}=0, n \geq 2$. We can verify the large- $n$ estimate (22) for the normalized eigenfunctions by expanding (24) in powers of $n^{-1}$.

Remark 4. The density (25) of Brownian motion with drift between two reflecting barriers has apparently remained unpublished until recently, while the driftless version of this formula is classic. The density (25) appeared recently in the currency exchange rate target-zone model in a preliminary version of Svensson (1991) (corrected in de Jong (1994)). Using Poisson's summation formula, it can alternatively be expressed as a series of terms involving Gaussian densities and cumulative distribution functions (Veestraeten (2004)).

\subsection{Brownian motion with drift on $[0, \infty)$, reflected at 0}

Now suppose that $\mu<0$. Infinity is a nonattracting natural boundary and $\int_{0}^{\infty} \mathfrak{m}(x) \mathrm{d} x=$ $1 /|\mu|$. The stationary density is exponential:

$$
\pi(x)=2|\mu| \mathrm{e}^{-2|\mu| x} .
$$

The Liouville transformation reduces the SL equation to the Schrödinger equation (27) with constant potential $Q=\frac{1}{2} \mu^{2}, x \in(0, \infty)$, and the boundary condition $v^{\prime}(0)-\gamma v(0)=0$, $\gamma=\mu / 2^{1 / 2}$. From (15), infinity is an oscillatory natural boundary with cutoff $\Lambda=\frac{1}{2} \mu^{2}$, while, from (16), the SL equation is nonoscillatory for $\lambda=\Lambda$. The SL operator has an absolutely continuous spectrum in $\left[\frac{1}{2} \mu^{2}, \infty\right)$ plus a single eigenvalue $\lambda_{0}=0$ in $\left[0, \frac{1}{2} \mu^{2}\right]$. We shall obtain the spectral representation of Brownian motion on $[0, \infty)$, reflected at 0 , by explicitly taking the limit as $r \uparrow \infty$ in (25). Introduce $\Delta s:=\pi / r$ and $s_{n}:=n \Delta s, n=1,2, \ldots$, and write (25) as follows:

$$
\begin{aligned}
p^{(r)}(t ; x, y)= & \frac{2 \mu \mathrm{e}^{2 \mu x}}{\mathrm{e}^{2 \mu r}-1}+\frac{2}{\pi} \mathrm{e}^{\mu(y-x)-\mu^{2} t / 2} \\
& \times \sum_{n=1}^{\infty} \frac{\mathrm{e}^{-\left(s_{n}^{2} / 2\right) t}}{s_{n}^{2}+\mu^{2}}\left\{s_{n} \cos \left(s_{n} x\right)+\mu \sin \left(s_{n} x\right)\right\}\left\{s_{n} \cos \left(s_{n} y\right)+\mu \sin \left(s_{n} y\right)\right\} \Delta s .
\end{aligned}
$$

Taking the limit as $r \uparrow \infty$ is equivalent to taking the limit as $\Delta s \downarrow 0$. The series in (28) has the form of a Riemannian sum and, in the limit, we obtain the following integral:

$$
\begin{aligned}
p(t ; x, y)= & 2|\mu| \mathrm{e}^{-2|\mu| y}+\frac{2}{\pi} \mathrm{e}^{\mu(y-x)-\mu^{2} t / 2} \\
& \times \int_{0}^{\infty} \frac{\mathrm{e}^{-\left(s^{2} t / 2\right)}}{s^{2}+\mu^{2}}\{s \cos (s x)+\mu \sin (s x)\}\{s \cos (s y)+\mu \sin (s y)\} \mathrm{d} s .
\end{aligned}
$$


For the cumulative distribution function, we similarly obtain

$$
\begin{aligned}
\mathrm{P}_{x}\left(X_{t} \leq y\right)= & 1-\mathrm{e}^{-2|\mu| y}+\frac{2}{\pi} \mathrm{e}^{\mu(y-x)-\mu^{2} t / 2} \\
& \times \int_{0}^{\infty} \frac{\mathrm{e}^{-s^{2} t / 2}}{s^{2}+\mu^{2}}\{s \cos (s x)+\mu \sin (s x)\} \sin (s y) \mathrm{d} s .
\end{aligned}
$$

The integral in (29) can be expressed in terms of the standard normal cumulative distribution function $\Phi(x)$ :

$$
\begin{gathered}
\frac{2}{\pi} \mathrm{e}^{\mu(y-x)-\mu^{2} t / 2} \int_{0}^{\infty} \frac{\mathrm{e}^{-s^{2} t / 2}}{s^{2}+\mu^{2}}\{s \cos (s x)+\mu \sin (s x)\} \sin (s y) \mathrm{d} s \\
=\mathrm{e}^{2 \mu y} \Phi((x+y+\mu t) / \sqrt{t})-\Phi((x-y+\mu t) / \sqrt{t}) .
\end{gathered}
$$

(The proof of this integral identity is available from the author upon request.) Substituting (30) into (29) yields the following well-known result for Brownian motion reflected at the origin (e.g. Harrison (1985, p. 49) and Abate and Whitt (1987a), (1987b)):

$$
\mathrm{P}_{x}\left(X_{t} \leq y\right)=\Phi((y-x-\mu t) / \sqrt{t})-\mathrm{e}^{2 \mu y} \Phi((-y-x-\mu t) / \sqrt{t}) .
$$

\section{Reflected Ornstein-Uhlenbeck process}

\subsection{OU process on $[0, r]$, reflected at 0 and $r$}

Consider an Ornstein-Uhlenbeck process with infinitesimal diffusion and drift parameters $a(x)=\sigma$ and $b(x)=\kappa(\theta-x)$ on $[0, r]$, reflected at 0 and $r$. Here $\theta \in(0, r)$ is the long-run level, $\kappa>0$ is the rate of mean reversion towards the long-run level, and $\sigma>0$ is the constant diffusion parameter (volatility). The scale and speed densities are

$$
\mathfrak{s}(x)=\mathrm{e}^{\kappa(\theta-x)^{2} / \sigma^{2}}, \quad \mathfrak{m}(x)=\frac{2}{\sigma^{2}} \mathrm{e}^{-\kappa(\theta-x)^{2} / \sigma^{2}} .
$$

The stationary density (1) is

$$
\pi(x)=\frac{\sqrt{2 \kappa}}{\sigma} \frac{\phi(z)}{\Phi(\beta)-\Phi(\alpha)},
$$

where $z \in[\alpha, \beta]$ is a standardized variable with

$$
z:=\frac{\sqrt{2 \kappa}}{\sigma}(x-\theta), \quad \alpha:=-\frac{\sqrt{2 \kappa}}{\sigma} \theta, \quad \beta:=\frac{\sqrt{2 \kappa}}{\sigma}(r-\theta),
$$

and $\phi(x)$ and $\Phi(x)$ are the standard normal density and cumulative distribution function, respectively.

The associated SL equation is

$$
\frac{1}{2} \sigma^{2} \frac{\mathrm{d}^{2} u}{\mathrm{~d} x^{2}}+\kappa(\theta-x) \frac{\mathrm{d} u}{\mathrm{~d} x}+\lambda u=0 .
$$

We introduce the standardized variable $z:=(2 \kappa)^{1 / 2}(x-\theta) / \sigma$ and look for solutions of the form $u(x)=\exp \left(\frac{1}{4} z^{2}\right) w(z)$. Substituting this functional form into (33), we arrive at the WeberHermite equation for $w$ (Erdelyi (1953, p. 116) and Buchholz (1969, p. 39)):

$$
\frac{\mathrm{d}^{2} w}{\mathrm{~d} z^{2}}+\left(\frac{1}{2}+v-\frac{1}{4} z^{2}\right) w=0, \quad \text { where } v:=\frac{\lambda}{\kappa} .
$$


For any $\lambda \in \mathbb{C}$, the pair of linearly independent solutions are provided by the Weber-Hermite parabolic cylinderfunctions $E_{v}^{(0)}(z)$ and $E_{v}^{(1)}(z)$ (Buchholz (1969, pp. 40-43)). These functions are related by (Buchholz (1969, p. 40))

$$
E_{v}^{(0)}(z)=\sqrt{2} \mathrm{e}^{-z^{2} / 4}{ }_{1} F_{1}\left(-\frac{1}{2} \nu ; \frac{1}{2} ; \frac{1}{2} z^{2}\right), \quad E_{v}^{(1)}(z)=2 z \mathrm{e}^{-z^{2} / 4}{ }_{1} F_{1}\left(\frac{1}{2}(1-v) ; \frac{3}{2} ; \frac{1}{2} z^{2}\right)
$$

to the Kummer confluent hypergeometric function ${ }_{1} F_{1}(a ; b ; x)$, which is defined for all $z \in \mathbb{C}$, $a \in \mathbb{C}$, and $b \in \mathbb{C} \backslash\{0,-1,-2, \ldots\}$ by the series

$$
{ }_{1} F_{1}(a ; b ; x)=\sum_{n=0}^{\infty} \frac{(a)_{n}}{(b)_{n}} \frac{z^{n}}{n !},
$$

where $(a)_{0}:=1,(a)_{n}:=a(a+1) \cdots(a+n-1)$ are the Pochhammer symbols. (The Kummer confluent hypergeometric function ${ }_{1} F_{1}(a ; b ; x)$ is available as a built-in function in both the MATHEMATICA and MAPLE ${ }^{\circledR}$ software packages (Hypergeometric1F1 $[a, b, z]$ in MATHEMATICA). To efficiently compute the function, depending on the values of $a, b$, and $z$, these software packages use several integral representations and asymptotic expansions in addition to the series (34) (see Slater (1960) and Buchholz (1969)).)

The Weber-Hermite parabolic cylinder functions have the differential properties

$$
\frac{\mathrm{d}}{\mathrm{d} z}\left\{\mathrm{e}^{z^{2} / 4} E_{\nu}^{(0)}(z)\right\}=-2^{-1 / 2} \nu \mathrm{e}^{z^{2} / 4} E_{v-1}^{(1)}(z), \quad \frac{\mathrm{d}}{\mathrm{d} z}\left\{\mathrm{e}^{z^{2} / 4} E_{v}^{(1)}(z)\right\}=2^{1 / 2} \mathrm{e}^{z^{2} / 4} E_{v-1}^{(0)}(z)
$$

and have a Wronskian independent of $z$ (Buchholz (1969, p. 43)):

$$
\begin{aligned}
E_{v}^{(0)}(z) \frac{\mathrm{d}}{\mathrm{d} z}\left\{E_{v}^{(1)}(z)\right\}-E_{v}^{(1)}(z) \frac{\mathrm{d}}{\mathrm{d} z}\left\{E_{v}^{(0)}(z)\right\} & =2^{-1 / 2}\left\{v E_{v}^{(1)}(z) E_{v-1}^{(1)}(z)+2 E_{v}^{(0)}(z) E_{v-1}^{(0)}(z)\right\} \\
& =2^{(v+3) / 2} .
\end{aligned}
$$

We furthermore introduce the function

$$
\Upsilon(\nu ; x, y):=v E_{v}^{(1)}(x) E_{\nu-1}^{(1)}(y)+2 E_{v}^{(0)}(x) E_{v-1}^{(0)}(y) .
$$

From (36), $\Upsilon(v ; x, x)=2^{v / 2+2}$.

Using (35)-(37), we obtain solutions $\phi(x, \lambda)$ and $\psi(x, \lambda)$, with the initial conditions

$$
\phi(r, \lambda)=1 \quad \text { and } \quad \phi^{\prime}(r, \lambda)=0, \quad \text { and } \quad \psi(0, \lambda)=1 \quad \text { and } \quad \psi^{\prime}(0, \lambda)=0,
$$

given by

$$
\phi(x, \lambda)=2^{-v / 2-2} \mathrm{e}^{\left(z^{2}-\beta^{2}\right) / 4} \Upsilon(\nu ; z, \beta), \quad \psi(x, \lambda)=2^{-v / 2-2} \mathrm{e}^{\left(z^{2}-\alpha^{2}\right) / 4} \Upsilon(v ; z, \alpha) .
$$

The Wronskian (11) is

$$
w(\lambda)=2^{-(\nu+3) / 2} \kappa^{1 / 2} \sigma^{-1} \mathrm{e}^{-\left(\alpha^{2}+\beta^{2}\right) / 4} \nu \Delta(\nu ; \alpha, \beta),
$$

where

$$
\Delta(\nu ; \alpha, \beta):=E_{\nu-1}^{(0)}(\alpha) E_{\nu-1}^{(1)}(\beta)-E_{\nu-1}^{(1)}(\alpha) E_{\nu-1}^{(0)}(\beta) .
$$

Recalling that $\nu=\lambda / \kappa$, the Wronskian zeros/eigenvalues are

$$
\lambda_{0}=0, \quad \lambda_{n}=\kappa v_{n}, \quad n=1,2, \ldots,
$$


where $\left\{v_{n}, n=1,2, \ldots\right\}$ are positive roots of the equation

$$
\Delta(\nu ; \alpha, \beta)=0 .
$$

The normalized eigenfunctions (9) are given by

$$
\begin{array}{ll}
\varphi_{0}(x)= \pm c^{-1 / 2}, & c=2 \pi^{1 / 2} \sigma^{-1} \kappa^{-1 / 2}[\Phi(\beta)-\Phi(\alpha)], \\
\varphi_{n}(x)= \pm \frac{2^{1 / 4} \kappa \mathrm{e}^{z^{2} / 4} \Upsilon\left(\nu_{n} ; z, \beta\right)}{\sqrt{\lambda_{n} \Delta_{n} \Upsilon\left(\nu_{n} ; \alpha, \beta\right)}}, & \Delta_{n}:=\left.\frac{\partial \Delta(\nu ; \alpha, \beta)}{\partial \nu}\right|_{\nu=v_{n}}, \quad n=1,2, \ldots
\end{array}
$$

To evaluate the derivative (40) of the function (38) with respect to $v$, we recall the derivative of the Kummer function with respect to its first index (this derivative is available in MATHEMATICA with the call Hypergeometric1F $\left.1^{(1,0,0)}[a, b, z]\right)$, where $\psi(z)=\Gamma^{\prime}(z) / \Gamma(z)$ is the digamma function (Abramowitz and Stegun (1972)):

$$
\frac{\partial}{\partial a}\left\{{ }_{1} F_{1}(a ; b ; z)\right\}=\sum_{k=0}^{\infty} \frac{(a)_{k} \psi(a+k)}{(b)_{k} k !} z^{k}-\psi(a)_{1} F_{1}(a ; b ; z) .
$$

Finally, substituting the eigenvalues and eigenfunctions into (8), we obtain the spectral representation for the reflected OU density.

The roots $v_{n}$ have to be found numerically. The large- $n$ estimates are given by (21). The Liouville transformation $y=2^{1 / 2} x / \sigma, u(x)=2^{-1 / 4} \sigma^{1 / 2} \exp \left(\kappa(\theta-x)^{2} / 2 \sigma^{2}\right) v(y(x))$ reduces the SL equation (33) to the Schrödinger equation with quadratic potential

$$
\frac{\mathrm{d}^{2} v}{\mathrm{~d} y^{2}}+[\lambda-Q(y)] v=0, \quad Q(y)=\frac{\kappa^{2}}{4}\left(y+\frac{\alpha}{\sqrt{\kappa}}\right)^{2}-\frac{\kappa}{2}, \quad y \in(0, R), R=\frac{\sqrt{2}}{\sigma} r
$$

subject to the boundary conditions (20) with $\gamma_{1}=\kappa \theta / \sigma 2^{1 / 2}$ and $\gamma_{2}=\kappa(\theta-r) / \sigma 2^{1 / 2}$. Specializing (21) to our case, we obtain

$$
\lambda_{n}=\frac{\sigma^{2} \pi^{2} n^{2}}{2 r^{2}}+a_{0}+O\left(\frac{1}{n^{2}}\right), \quad a_{0}=\frac{\kappa}{2}+\frac{\kappa^{2}}{6 \sigma^{2}}\left(r^{2}-3 r \theta+3 \theta^{2}\right) .
$$

The estimate (22) for the eigenfunctions gives the following estimate for the eigenfunctions (40):

$$
\begin{gathered}
\varphi_{n}(x)= \pm \sigma r^{-1 / 2} \mathrm{e}^{z^{2} / 4}\left\{\cos \left(\frac{n \pi x}{r}\right)+\frac{r}{n \pi \sigma^{2}} f(x) \sin \left(\frac{n \pi x}{r}\right)\right\}+O\left(\frac{1}{n^{2}}\right), \\
f(x)=\frac{\kappa^{2}}{6 \sigma^{2}} x^{3}-\frac{\kappa^{2} \theta}{2 \sigma^{2}} x^{2}+\left(\frac{\kappa}{2}\left(\frac{\alpha}{2}-1\right)-a_{0}\right) x+\kappa \theta .
\end{gathered}
$$

\subsection{OU process on $[0, \infty)$, reflected at 0}

Consider the OU process on $[0, \infty)$, reflected at 0 . Infinity is a nonattracting natural boundary. The stationary density is

$$
\pi(x)=\frac{\sqrt{2 \kappa}}{\sigma} \frac{\phi(z)}{1-\Phi(\alpha)}
$$

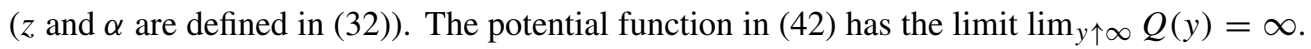
Hence, infinity is nonoscillatory and the spectrum is purely discrete. 
The unique (up to a multiple independent of $x$ ) solution $\phi(x, \lambda)$ that is square integrable with weight $\mathfrak{m}(x)$ for each $\lambda \in \mathbb{C}$, i.e. $\int_{0}^{\infty}|\phi(x, \lambda)|^{2} \mathfrak{m}(x) \mathrm{d} x<\infty$, and entire in $\lambda$ for each $x \in[0, \infty)$ can be written in the form

$$
\phi(x, \lambda)=H_{v}\left(2^{-1 / 2} z\right), \quad v=\lambda / \kappa
$$

(recall that $\left.z=(2 \kappa)^{1 / 2}(x-\theta) / \sigma\right)$, where $H_{v}(z)$ is the Hermite function, defined by (Lebedev (1972, p. 285))

$$
H_{v}(z)=2^{v} \sqrt{\pi}\left\{\frac{{ }_{1} F_{1}\left(-\frac{1}{2} v ; \frac{1}{2} ; z^{2}\right)}{\Gamma\left(\frac{1}{2}(1-v)\right)}-2 z \frac{{ }_{1} F_{1}\left(\frac{1}{2}(1-v) ; \frac{3}{2} ; z^{2}\right)}{\Gamma\left(-\frac{1}{2} v\right)}\right\} .
$$

(The Hermite function is available in MATHEMATICA with the call HermiteH $[v, z]$.) The solution $\phi(x, \lambda)$ has the following asymptotic properties:

$$
\lim _{x \uparrow \infty} \phi(x, \lambda)=0, \quad \lim _{x \uparrow \infty} \frac{\phi^{\prime}(x, \lambda)}{\mathfrak{s}(x)}=0 .
$$

Using the differential property

$$
\frac{\mathrm{d}}{\mathrm{d} z}\left\{H_{v}(z)\right\}=2 v H_{v-1}(z)
$$

the Wronskian is

$$
w(\lambda)=2 \lambda \sigma^{-1} \kappa^{-1 / 2} \mathrm{e}^{-\alpha^{2} / 2} H_{\nu-1}\left(2^{-1 / 2} \alpha\right)
$$

The eigenvalues are

$$
\lambda_{0}=0, \quad \lambda_{n}=\kappa v_{n}, \quad n=1,2, \ldots,
$$

where $v_{n}$ are roots of the equation

$$
H_{\nu-1}\left(2^{-1 / 2} \alpha\right)=0
$$

The normalized eigenfunctions are given by

$$
\begin{array}{ll}
\varphi_{0}(x)= \pm c^{-1 / 2}, & c=2 \pi^{1 / 2} \sigma^{-1} \kappa^{-1 / 2}[1-\Phi(\alpha)], \\
\varphi_{n}(x)= \pm \frac{\kappa^{3 / 4} \sigma^{1 / 2} \mathrm{e}^{\alpha^{2} / 4} H_{\nu_{n}}\left(2^{-1 / 2} z\right)}{\sqrt{2 \lambda_{n} \Delta_{n} H_{v_{n}}\left(2^{-1 / 2} \alpha\right)}}, & \Delta_{n}:=\left.\frac{\partial H_{\nu-1}\left(2^{-1 / 2} \alpha\right)}{\partial \nu}\right|_{\nu=v_{n}}, \quad n=1,2, \ldots
\end{array}
$$

To calculate the derivative of the Hermite function with respect to its index, recall (45) and (41) (this derivative is available in MATHEMATICA with the call $\operatorname{HermiteH}^{(1,0)}[v, z]$ ).

To determine the eigenvalues $\lambda_{n}, n \geq 1$, we must find the roots of (47) numerically. A useful estimate for the eigenvalues can be obtained as follows. The Hermite function can be expressed in terms of the Tricomi confluent hypergeometric function $U(a, b, z)$ (Lebedev (1972, p. 293)):

$$
H_{v}(x)=2^{v} U\left(-\frac{1}{2} v, \frac{1}{2}, x^{2}\right) .
$$

Using an estimate of the function $U(a, b, z)$ for $a \rightarrow-\infty$ (Slater (1960, p. 69, Equation (4.4.18))),

$U(a, b, z)=2^{1 / 2} z^{1 / 4-b / 2} \mathrm{e}^{z / 2} k^{k-1 / 4} \mathrm{e}^{-k} \cos \left(2 \sqrt{k z}-\pi k+\frac{1}{4} \pi\right)\left\{1+O\left(k^{-1 / 2}\right)\right\}, \quad k=\frac{1}{2} b-a$, 
we have an estimate of the Hermite function for $v \rightarrow \infty$ :

$$
H_{\nu}(x)=2^{\nu+1 / 2} \mathrm{e}^{x^{2} / 2} k^{k-1 / 4} \mathrm{e}^{-k} \cos \left(2 x \sqrt{k}-\pi k+\frac{1}{4} \pi\right)\left\{1+O\left(k^{-1 / 2}\right)\right\}, \quad k=\frac{1}{2} \nu+\frac{1}{4} .
$$

From (48), (46), and (47), for large $n$ the eigenvalues $\lambda_{n}$ can be estimated as follows:

$$
\lambda_{n} \sim 2 \kappa n+\frac{2 \kappa \alpha^{2}}{\pi^{2}}+\frac{2^{3 / 2} \kappa \alpha}{\pi} \sqrt{n-\frac{1}{4}+\frac{\alpha^{2}}{2 \pi^{2}}} .
$$

Note that, for the process on the infinite interval $[0, \infty)$, the eigenvalues $\lambda_{n}$ grow linearly with $n$, in contrast with the $n^{2}$ growth, in (43), for the process on the finite interval. We also note that, the larger the mean-reversion parameter $\kappa$, the faster the eigenvalue growth.

Remark 5. The Laplace transform of the transition density for the reflected OU process on $[0, \infty)$ was previously obtained in Ricciardi and Sacerdote (1987) in the context of applications in mathematical biology. The spectral expansion obtained in this section can be regarded as the Laplace inversion of their transform $\left(\lambda_{n}\right.$ are the poles of the Laplace transform and $\mathfrak{m}(y) \varphi_{n}(x) \varphi_{n}(y)$ are the corresponding residues). An analytical approximation of the transition density for small $\kappa$ was recently obtained in Ward and Glynn (2003b), in the context of queueing applications.

\section{Reflected affine diffusion}

\subsection{Affine diffusion on $[0, r]$, reflected at 0 and $r$}

Consider an affine diffusion with infinitesimal parameters $a(x)=\sigma(x-\ell)^{1 / 2}$ and $b(x)=$ $\kappa(\theta-x)$ on $[0, r]$, reflected at 0 and $r$. Here, $\theta \in(0, r)$ is the long-run level, $\kappa>0$ is the rate of mean reversion towards the long-run level, $\sigma>0$ is the volatility, and $\ell<0$ is the shift parameter. This process is a shifted square-root diffusion of Feller (1951) restricted to the interval $[0, r]$, with reflection at the boundaries (see also Shiga and Watanabe (1973), Pitman and Yor (1982), and Göing-Jaeschke and Yor (2003) for more details on square-root processes; this process is known as a Cox-Ingersoll-Ross model (Cox et al. (1985)) in the finance literature). The scale and speed densities are

$$
\mathfrak{s}(x)=(x-\ell)^{-b} \mathrm{e}^{2 \kappa(x-\ell) / \sigma^{2}}, \quad \mathfrak{m}(x)=\frac{2}{\sigma^{2}}(x-\ell)^{b-1} \mathrm{e}^{-2 \kappa(x-\ell) / \sigma^{2}},
$$

where

$$
b:=\frac{2 \kappa(\theta-\ell)}{\sigma^{2}}>0 .
$$

The stationary density is

$$
\pi(x)=c^{-1} z^{b-1} \mathrm{e}^{-z}, \quad c=\frac{\sigma^{2}}{2 \kappa}[\gamma(b, \beta)-\gamma(b, \alpha)],
$$

where $z \in[\alpha, \beta]$ is a standardized variable,

$$
z:=\frac{2 \kappa(x-\ell)}{\sigma^{2}}, \quad \alpha:=-\frac{2 \kappa \ell}{\sigma^{2}}, \quad \beta:=\frac{2 \kappa(r-\ell)}{\sigma^{2}},
$$

and $\gamma(b, x)=\int_{0}^{x} z^{b-1} \mathrm{e}^{-z} \mathrm{~d} x$ is the incomplete gamma function (see Abramowitz and Stegun (1972)). 
The associated SL equation is

$$
\frac{1}{2} \sigma^{2}(x-\ell) \frac{\mathrm{d}^{2} u}{\mathrm{~d} x^{2}}+\kappa(\theta-x) \frac{\mathrm{d} u}{\mathrm{~d} x}+\lambda u=0, \quad x \in[0, r] .
$$

Let $z=z(x)$ be the standardized variable defined in (52). Substituting $u(x)=w(z(x))$ into (53), we arrive at the confluent hypergeometric equation for $w=w(z)$ (Slater (1960, p. 2)),

$$
z \frac{\mathrm{d}^{2} w}{\mathrm{~d} z^{2}}+(b-z) \frac{\mathrm{d} w}{\mathrm{~d} z}-a w=0, \quad a:=-\frac{\lambda}{\kappa}, \quad z \in[\alpha, \beta],
$$

where $b$ is as defined in (50) and $\alpha$ and $\beta$ are as defined in (52). For any $a \in \mathbb{C}$, the pair of linearly independent solutions are provided by $U(a, b, z)$ and $\mathrm{e}^{z} U(b-a, b,-z)$, where $U(a, b, z)$ is the Tricomi confluent hypergeometric function defined for all $a, z \in \mathbb{C}$ and $b \in \mathbb{C} \backslash\{0, \pm 1, \pm 2, \ldots\}$ by (Slater (1960, p. 5))

$$
U(a, b, x)=\frac{\Gamma(1-b)}{\Gamma(1+a-b)}{ }_{1} F_{1}(a ; b ; x)+\frac{\Gamma(b-1)}{\Gamma(a)} x^{1-b}{ }_{1} F_{1}(1+a-b ; 2-b ; x) .
$$

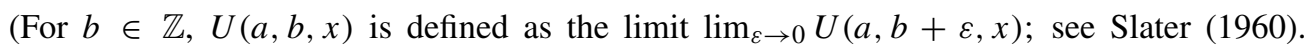
The Tricomi confluent hypergeometric function is available in MATHEMATICA with the call Hypergeometric $\mathrm{U}[a, b, z]$.)

These solutions have the differential properties (Slater (1960, p. 16))

$$
\begin{aligned}
\frac{\mathrm{d}}{\mathrm{d} z}\{U(a, b, z)\} & =-a U(a+1, b+1, z), \\
\frac{\mathrm{d}}{\mathrm{d} z}\left\{\mathrm{e}^{z} U(b-a, b,-z)\right\} & =\mathrm{e}^{z} U(b-a, b+1,-z),
\end{aligned}
$$

and the Wronskian (Slater (1960, p. 18))

$$
U(a, b, z) \frac{\mathrm{d}}{\mathrm{d} z}\left\{\mathrm{e}^{z} U(b-a, b,-z)\right\}-\mathrm{e}^{z} U(b-a, b,-z) \frac{\mathrm{d}}{\mathrm{d} z}\{U(a, b, z)\}=\mathrm{e}^{\mathrm{i} \pi(a-b)} z^{-b} \mathrm{e}^{z}
$$

We introduce the following notation:

$$
\begin{aligned}
\Xi(a, b ; x, y):= & \mathrm{e}^{y} U(a, b, x) U(b-a, b+1,-y) \mathrm{e}^{\mathrm{i} \pi(b-a)} \\
& +a \mathrm{e}^{x} U(a+1, b+1, y) U(b-a, b,-x) \mathrm{e}^{\mathrm{i} \pi(b-a)} .
\end{aligned}
$$

From (55)-(57), $\Xi(a, b ; z, z)=z^{-b} \mathrm{e}^{z}$. Using (55)-(58), we obtain solutions $\phi(x, \lambda)$ and $\psi(x, \lambda)$, with the initial conditions $\phi(r, \lambda)=1$ and $\phi^{\prime}(r, \lambda)=0$, and $\psi(0, \lambda)=1$ and $\psi^{\prime}(0, \lambda)=0$, given by

$$
\phi(x, \lambda)=\beta^{b} \mathrm{e}^{-\beta} \Xi(a, b ; z, \beta), \quad \psi(x, \lambda)=\alpha^{b} \mathrm{e}^{-\alpha} \Xi(a, b ; z, \alpha) .
$$

The Wronskian (11) is

$$
w(\lambda)=a\left(\frac{2 \kappa}{\sigma^{2}}\right)^{1-b}(\alpha \beta)^{b} \mathrm{e}^{-\alpha-\beta} \Delta(a, b ; \alpha, \beta),
$$

where

$$
\begin{aligned}
\Delta(a, b ; \alpha, \beta)= & \mathrm{e}^{\alpha} U(a+1, b+1, \beta) U(b-a, b+1,-\alpha) \mathrm{e}^{\mathrm{i} \pi(b-a)} \\
& -\mathrm{e}^{\beta} U(a+1, b+1, \alpha) U(b-a, b+1,-\beta) \mathrm{e}^{\mathrm{i} \pi(b-a)} .
\end{aligned}
$$


Recalling that $a=-\lambda / \kappa$, the Wronskian zeros/eigenvalues are

$$
\lambda_{0}=0, \quad \lambda_{n}=-\kappa a_{n}, \quad n=1,2, \ldots,
$$

where $0>a_{1}>a_{2}>\cdots$ are negative roots of the equation

$$
\Delta(a, b ; \alpha, \beta)=0
$$

(for fixed $b, \alpha$, and $\beta$ ). The normalized eigenfunctions (9) are given by

$$
\begin{array}{ll}
\varphi_{0}(x)= \pm c^{-1 / 2} & (c \text { is as defined in }(51)), \\
\varphi_{n}(x)= \pm\left(\frac{2 \kappa}{\sigma^{2}}\right)^{b / 2} \frac{\kappa^{1 / 2} \sigma \Xi\left(a_{n}, b ; z, \beta\right)}{\sqrt{2 \lambda_{n} \Delta_{n} \alpha^{b} \mathrm{e}^{-\alpha} \Xi\left(a_{n}, b ; \alpha, \beta\right)}}, \quad \Delta_{n}:=\left.\frac{\partial \Delta(a, b ; \alpha, \beta)}{\partial a}\right|_{a=a_{n}}, \quad n \geq 1 .
\end{array}
$$

To compute the derivative with respect to $a$, recall (59) and the derivative of $U(a, b, z)$ with respect to its first index $a$ (this derivative is available in MATHEMATICA with the call Hypergeometric $\left.^{(1,0,0)}[a, b, z]\right)$ :

$$
\begin{aligned}
\frac{\partial}{\partial a}\{U(a, b, z)\}= & \frac{\Gamma(b-1) z^{1-b}}{\Gamma(a)} \sum_{k=0}^{\infty} \frac{\psi(a-b+k+1)(a-b+1)_{k} z^{k}}{k !(2-b)_{k}} \\
& +\frac{\Gamma(1-b)}{\Gamma(a-b+1)} \sum_{k=0}^{\infty} \frac{\psi(a+k)(a)_{k} z^{k}}{k !(b)_{k}} \\
& -\{\psi(a)+\psi(a-b+1)\} U(a, b, z) .
\end{aligned}
$$

The eigenvalues $\lambda_{n}$ have to be found numerically by finding the roots $a_{n}$ of (60). The large- $n$ estimates are given by (21). The Liouville transformation

$$
y=\frac{2^{3 / 2}}{\sigma}(\sqrt{x-\ell}-\sqrt{-\ell}), \quad u(x)=2^{-1 / 4} \sigma^{1 / 2}(x-\ell)^{-b / 2+1 / 4} \mathrm{e}^{\kappa(x-\ell) / \sigma^{2}} v(y(x))
$$

reduces the SL equation (54) to the Schrödinger equation

$$
\frac{\mathrm{d}^{2} v}{\mathrm{~d} y^{2}}+[\lambda-Q(y)] v=0, \quad y \in(0, R), R=2^{3 / 2} \sigma^{-1}(\sqrt{r-\ell}-\sqrt{-\ell}),
$$

with potential

$$
Q(y)=\frac{\frac{3}{4}+b(b-2)}{(y+a)^{2}}-\frac{1}{2} \kappa b+\frac{1}{16} \kappa^{2}(y+a)^{2}, \quad a=2^{3 / 2} \sigma^{-1} \sqrt{-\ell}
$$

and boundary conditions (20) with

$$
\gamma_{1}=\sqrt{\frac{\kappa}{\alpha}}\left(\frac{\kappa \theta}{\sigma^{2}}-\frac{1}{2}\right), \quad \gamma_{2}=\sqrt{\frac{\kappa}{\beta}}\left(\frac{\kappa(\theta-r)}{\sigma^{2}}-\frac{1}{2}\right) .
$$

Specializing (21) to our case, we obtain

$$
\begin{gathered}
\lambda_{n}=\frac{\sigma^{2} n^{2} \pi^{2}}{8(\sqrt{r-\ell}-\sqrt{-\ell})^{2}}+a_{0}+O\left(\frac{1}{n^{2}}\right) \\
a_{0}=\frac{1}{12} \kappa(\beta+\alpha+\sqrt{\alpha \beta})-\frac{1}{2} \kappa b+\frac{\kappa}{\sqrt{\alpha \beta}}\left(\frac{1}{4} b(b-2)+\frac{\kappa \theta}{\sigma^{2}}-\frac{5}{16}\right)+\frac{\kappa^{2} r}{\sigma^{2}(\beta-\sqrt{\alpha \beta})} .
\end{gathered}
$$

Equation (22) gives the estimates for the eigenfunctions. 


\subsection{Affine diffusion on $[0, \infty)$, reflected at 0}

Consider an affine diffusion process on $[0, \infty)$, reflected at 0 . Infinity is a nonattracting natural boundary. The stationary density is

$$
\pi(x)=c^{-1} z^{b-1} \mathrm{e}^{-z}, \quad c=\frac{\sigma^{2}}{2 \kappa}[\Gamma(b)-\gamma(b, \alpha)],
$$

where $\Gamma(b)$ is the standard gamma function. The potential function in (62) has the limit $\lim _{y \uparrow \infty} Q(y)=\infty$. Hence, infinity is nonoscillatory and the spectrum is purely discrete.

The unique (up to a multiple independent of $x$ ) solution $\phi(x, \lambda)$ that is square integrable with weight $\mathfrak{m}(x)$ for each $\lambda \in \mathbb{C}$, i.e. $\int_{0}^{\infty}|\phi(x, \lambda)|^{2} \mathfrak{m}(x) \mathrm{d} x<\infty$, and entire in $\lambda$ for each $x \in[0, \infty)$ can be written in the form

$$
\phi(x, \lambda)=U(a, b, z)
$$

(recall that $\left.z=2 \kappa(x-\ell) / \sigma^{2}\right)$. This solution has the following asymptotic properties:

$$
\lim _{x \uparrow \infty} \phi(x, \lambda)=0, \quad \lim _{x \uparrow \infty} \frac{\phi^{\prime}(x, \lambda)}{\mathfrak{s}(x)}=0 .
$$

Using the differential property (57), the Wronskian is

$$
w(\lambda)=-a\left(\frac{2 \kappa}{\sigma^{2}}\right)^{1-b} \alpha^{b} \mathrm{e}^{-\alpha} U(a+1, b+1, \alpha) .
$$

The eigenvalues are

$$
\lambda_{0}=0, \quad \lambda_{n}=-\kappa a_{n}, \quad n=1,2, \ldots,
$$

where $a_{n}$ are the negative roots of the equation

$$
U(a+1, b+1, \alpha)=0 .
$$

The normalized eigenfunctions are given by

$$
\begin{aligned}
& \varphi_{0}(x)= \pm c^{-1 / 2} \quad(c \text { is as defined in (64)), } \\
& \varphi_{n}(x)= \pm\left(\frac{2 \kappa}{\sigma^{2}}\right)^{b / 2} \frac{\kappa^{1 / 2} \sigma U\left(a_{n}, b, z\right)}{\sqrt{2 \lambda_{n} \Delta_{n} \alpha^{b} \mathrm{e}^{-\alpha} U\left(a_{n}, b, \alpha\right)}}, \\
& \Delta_{n}:=-\left.\frac{\partial}{\partial a}\{U(a+1, b+1, \alpha)\}\right|_{a=a_{n}}, \quad n \geq 1 .
\end{aligned}
$$

To determine the eigenvalues, we need to find the roots of (65) numerically. Recalling the estimate (48), we obtain a useful estimate for the eigenvalues:

$$
\lambda_{n} \sim \kappa\left(n-\frac{1}{2} b+\frac{1}{4}+\frac{2 \alpha}{\pi^{2}}\right)+\frac{2 \kappa}{\pi} \sqrt{\left(n-\frac{1}{4}\right) \alpha+\frac{\alpha^{2}}{\pi^{2}}} .
$$

Note that, for the process on the infinite interval $[0, \infty)$, the eigenvalues $\lambda_{n}$ grow linearly with $n$, in contrast with the $n^{2}$ growth in (63) for the process on the finite interval. We also note that, the larger the mean-reversion parameter $\kappa$, the faster the eigenvalue growth. 


\section{Numerical examples}

\subsection{OU process}

Consider an OU process on [0, 2] with parameters $\sigma=1, \kappa=1$, and $\theta=1$, reflected at 0 and 2, and starting at the origin, $x=0$. To determine the eigenvalues $\lambda_{n}$ with $n \geq 1$, we use the root finding function FindRoot in MATHEMATICA to determine the roots $v_{n}$ of (39) (for fixed $\alpha$ and $\beta$ ). (The author used MATHEMATICA 4.0 running on a Pentium ${ }^{\circledR}$ III personal computer for all calculations in this paper.) For each $n \geq 1$, we use the estimate (43) as a starting point for the FindRoot function. Table 1 gives the eigenvalues $\lambda_{n}, n=1,2, \ldots, 10$, as well as the estimates (43). MATHEMATICA allows us to compute the eigenvalues to any desired accuracy. Table 1 shows that the estimates are accurate even for the lower eigenvalues, and that the accuracy of the estimates rapidly increases with $n$.

In Figure 1, we plot transition densities with $t=\frac{1}{4}, \frac{1}{2}, 1,2$, as well as the stationary density (31) (it was produced with the Plot function in MATHEMATICA). The stationary density is

TABLE 1: Eigenvalues of the OU process on [0,2], reflected at 0 and 2. The eigenvalues $\lambda_{n}$ are obtained by numerical root finding, and the estimates are obtained using (43). The OU process parameters are $\sigma=\kappa=\theta=1$ and $r=2$.

\begin{tabular}{rcc}
\hline$n$ & Eigenvalue $\lambda_{n}$ & Estimate \\
\hline 1 & 1.79846 & 1.90037 \\
2 & 5.57558 & 5.60147 \\
3 & 11.7588 & 11.7700 \\
4 & 20.3997 & 20.4059 \\
5 & 31.5053 & 31.5092 \\
6 & 45.0772 & 45.0799 \\
7 & 61.1160 & 61.1180 \\
8 & 79.6220 & 79.6235 \\
9 & 100.595 & 100.596 \\
10 & 124.036 & 124.037 \\
\hline
\end{tabular}

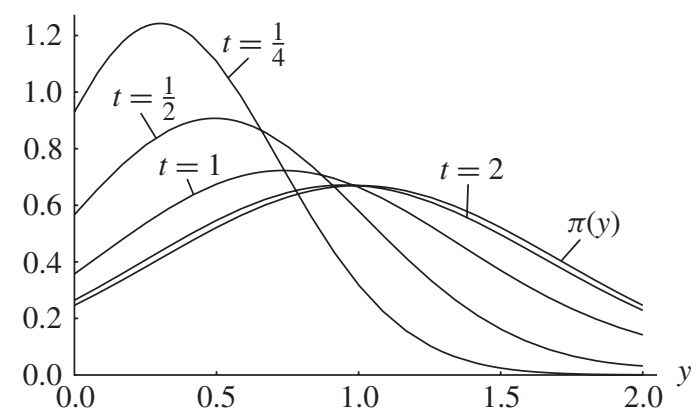

Figure 1: Transition densities $p(t ; 0, y)$, with $t=\frac{1}{4}, \frac{1}{2}, 1,2$, and the stationary density $\pi(y)$ for the OU process on $[0,2]$, reflected at 0 and 2 . The OU process parameters are $\sigma=\kappa=\theta=1$ and $r=2$, starting at the origin. 
TABLE 2: Eigenvalues of the OU process on $[0, \infty)$, reflected at 0 . The eigenvalues $\lambda_{n}$ are obtained by numerical root finding and the estimates are computed using (49). The OU process parameters are $\sigma=\kappa=\theta=1$ and $r=2$.

\begin{tabular}{rcc}
\hline$n$ & Eigenvalue $\lambda_{n}$ & Estimate \\
\hline 1 & 1.23423 & 1.23050 \\
2 & 2.69746 & 2.67287 \\
3 & 4.28019 & 4.25531 \\
4 & 5.92981 & 5.90658 \\
5 & 7.62244 & 7.60088 \\
6 & 9.34549 & 9.32538 \\
7 & 11.0915 & 11.0726 \\
8 & 12.8555 & 12.8376 \\
9 & 14.6342 & 14.6172 \\
10 & 16.4251 & 16.4090 \\
\hline
\end{tabular}

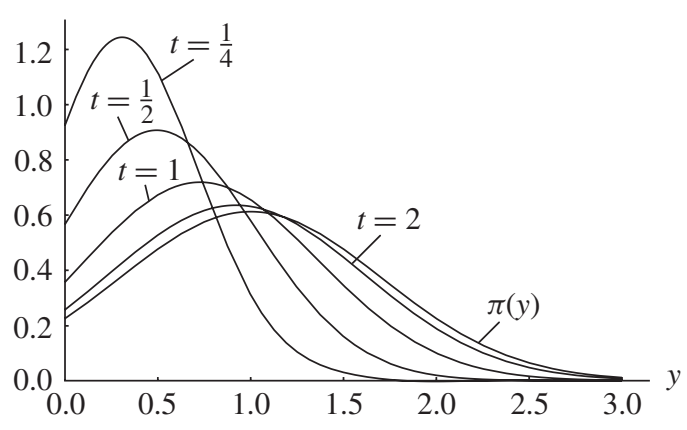

Figure 2: Transition densities $p(t ; 0, y)$, with $t=\frac{1}{4}, \frac{1}{2}, 1,2$, and the stationary density $\pi(y)$ for the OU process on $[0, \infty)$, reflected at 0 . The OU process parameters are $\sigma=\kappa=\theta=1$, starting at the origin.

the first term in the spectral expansion, corresponding to the principal eigenvalue $\lambda_{0}=0$. The terms in the series corresponding to the higher eigenvalues $\lambda_{n}$ are suppressed by the factors $\mathrm{e}^{-\lambda_{n} t}$. As $t$ increases, the transition density approaches the stationary density. The $t=2$ density is already very close to the stationary density. The spectral expansion method produces the transition density in a form especially convenient when studying how the system approaches its steady state.

Next consider the OU process on $[0, \infty$ ), reflected at 0 (the parameters are the same as before and the process starts at the origin). To determine $\lambda_{n}, n \geq 1$, we use the FindRoot function to determine the roots $v_{n}$ of (47) (for fixed $\alpha$ ). For each $n \geq 1$, we use the estimate (49) as a starting point for the FindRoot function. Table 2 gives the eigenvalues $\lambda_{n}, n=1,2, \ldots, 10$, as well as the estimates (49). In Figure 2, we plot transition densities with $t=\frac{1}{4}, \frac{1}{2}, 1,2$, as well as the stationary density (44). To further illustrate the convergence of eigenfunction expansions, in Table 3 we compute the mean $\mathrm{E}\left[X_{1} \mid X_{0}=0\right]$ and second moment $\mathrm{E}\left[X_{1}^{2} \mid X_{0}=0\right]$ of the OU process on $[0, \infty)$, reflected at 0 . The expectations are computed by integrating against the transition density (using the NIntegrate function in MATHEMATICA) and truncating the eigenfunction expansion after the $n$th term (the row with $n=0$ gives the steady-state values). 
TABLE 3: Mean $\mathrm{E}\left[X_{1} \mid X_{0}=0\right]$ and second moment $\mathrm{E}\left[X_{1}^{2} \mid X_{0}=0\right]$, at time $t=1$, of the OU process on $[0, \infty)$, reflected at 0 . The row with $n=0$ gives the steady-state values. The row with $n=1$ gives the values computed by truncating the eigenfunction expansion after the term with $n=1$, and similarly for the other rows. The OU process parameters are $\sigma=\kappa=\theta=1$, starting at the origin.

\begin{tabular}{ccc}
\hline$n$ & $\mathrm{E}\left[X_{1} \mid X_{0}=0\right]$ & $\mathrm{E}\left[X_{1}^{2} \mid X_{0}=0\right]$ \\
\hline 0 & 1.11264 & 1.61264 \\
1 & 0.89196 & 1.03627 \\
2 & 0.88515 & 1.05579 \\
3 & 0.88449 & 1.05637 \\
4 & 0.88442 & 1.05641 \\
5 & 0.88441 & 1.05641 \\
6 & 0.88441 & 1.05641 \\
\hline
\end{tabular}

Generally, owing to the presence of the factors $\mathrm{e}^{-\lambda_{n} t}$, the larger the time $t$, the faster the eigenfunction expansion converges.

\subsection{Affine process}

Consider an affine process on [0,2] with parameters $\sigma=1, \kappa=1, \theta=1$, and $\ell=-1$, reflected at 0 and 2 and starting at the origin, $x=0$. To determine the eigenvalues $\lambda_{n}, n \geq 1$, we use the FindRoot function in MATHEMATICA to determine the roots $v_{n}$ of (60) (for fixed $b, \alpha$, and $\beta$ ). For each $n \geq 1$, we use the estimate (63) as a starting point for the FindRoot function. Table 4 gives $\lambda_{n}, n=1,2, \ldots, 10$, as well as the estimates (63). MATHEMATICA allows us to compute the eigenvalues to any desired accuracy. Table 4 shows that the estimates are quite accurate even for the lower eigenvalues, and that the accuracy of the estimates rapidly increases with $n$. In Figure 3, we plot transition densities with $t=\frac{1}{4}, \frac{1}{2}, 1$, as well as the stationary density (51). As $t$ increases, the density approaches the stationary density; for $t=1$, the density is already quite close to the stationary density.

Next consider an affine process on $[0, \infty)$, reflected at 0 (the parameters are the same as before and the process starts at the origin). To determine $\lambda_{n}, n \geq 1$, we use the FindRoot

TABLE 4: Eigenvalues of the affine process on [0,2], reflected at 0 and 2. The eigenvalues $\lambda_{n}$ are obtained by numerical root finding, and the estimates are obtained using (63). The affine process parameters are $\sigma=\kappa=\theta=1, \ell=-1$, and $r=2$.

\begin{tabular}{rcc}
\hline$n$ & Eigenvalue $\lambda_{n}$ & \multicolumn{1}{c}{ Estimate } \\
\hline 1 & 2.82953 & 2.82195 \\
2 & 9.78285 & 9.72830 \\
3 & 21.3032 & 21.2389 \\
4 & 37.4214 & 37.3537 \\
5 & 58.1421 & 58.0727 \\
6 & 83.4662 & 83.3960 \\
7 & 113.394 & 113.324 \\
8 & 147.926 & 147.855 \\
9 & 187.063 & 186.991 \\
10 & 230.803 & 230.731 \\
\hline
\end{tabular}




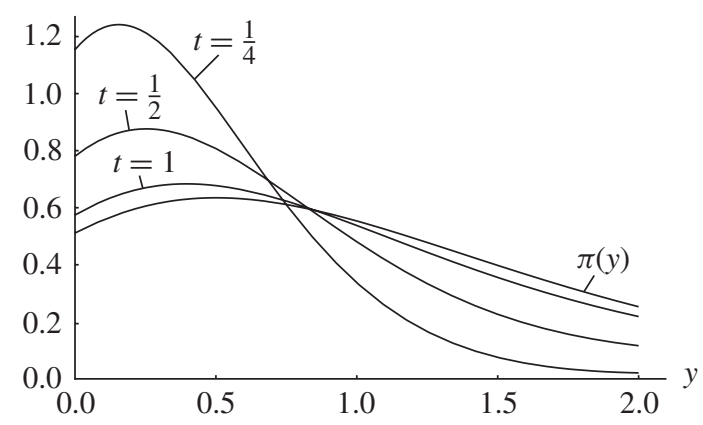

FIGURE 3: Transition densities $p(t ; 0, y)$, with $t=\frac{1}{4}, \frac{1}{2}, 1$, and the stationary density $\pi(y)$ for the affine process on $[0,2]$, reflected at 0 and 2 . The affine process parameters are $\sigma=\kappa=\theta=1, \ell=-1$, and $r=2$, starting at the origin.

TABLE 5: Eigenvalues of the affine process on $[0, \infty)$, reflected at 0 . The eigenvalues $\lambda_{n}$ are obtained by numerical root finding, and the estimates are obtained using (66). The affine process parameters are

$$
\sigma=\kappa=\theta=1 \text { and } \ell=-1 \text {. }
$$

\begin{tabular}{rcc}
\hline$n$ & Eigenvalue $\lambda_{n}$ & Estimate \\
\hline 1 & 1.21224 & 0.53402 \\
2 & 2.43540 & 1.91336 \\
3 & 3.64208 & 3.20232 \\
4 & 4.83223 & 4.44523 \\
5 & 6.00842 & 5.65890 \\
6 & 7.17303 & 6.85188 \\
7 & 8.32793 & 8.02923 \\
8 & 9.47459 & 9.19422 \\
9 & 10.6142 & 10.3491 \\
10 & 11.7476 & 11.4956 \\
100 & 107.737 & 107.656 \\
\hline
\end{tabular}

function to determine the roots $a_{n}$ of (65) (for fixed $b$ and $\alpha$ ). Table 5 gives the eigenvalues $\lambda_{n}, n=1,2, \ldots, 10$, as well as the estimates (66). In Figure 4, we plot transition densities with $t=\frac{1}{4}, \frac{1}{2}, 1,2$, as well as the stationary density (64). To further illustrate the convergence of eigenfunction expansions, in Table 6 we compute the mean $\mathrm{E}\left[X_{1} \mid X_{0}=0\right]$ and second moment $\mathrm{E}\left[X_{1}^{2} \mid X_{0}=0\right]$ of the affine diffusion on $[0, \infty)$, reflected at 0 . The expectations are computed by integrating against the transition density (using the NIntegrate function in MATHEMATICA) and truncating the eigenfunction expansion after the $\lambda_{n}$ term (the row with $n=0$ gives the steady-state values).

\section{Acknowledgements}

The author thanks Peter Glynn for stimulating discussions and comments on the earlier versions of this paper. This research was supported by the US National Science Foundation under grants DMI-0200429 and DMS-0223354. 


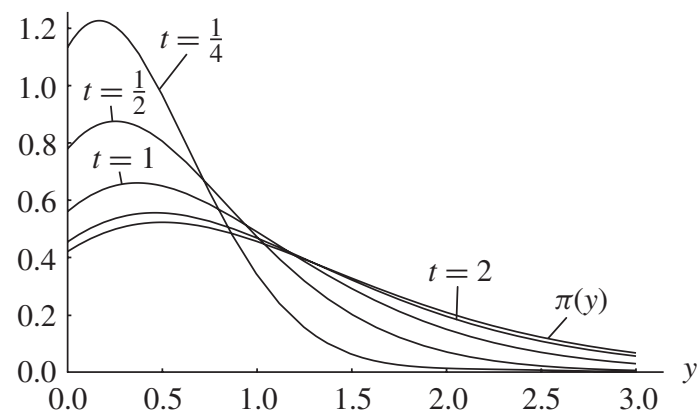

Figure 4: Transition densities $p(t ; 0, y)$, with $t=\frac{1}{4}, \frac{1}{2}, 1,2$, and the stationary density $\pi(y)$ for the affine process on $[0, \infty)$, reflected at 0 . The affine process parameters are $\sigma=\kappa=\theta=1$ and $\ell=-1$, starting at the origin.

TABle 6: Mean $\mathrm{E}\left[X_{1} \mid X_{0}=0\right]$ and second moment $\mathrm{E}\left[X_{1}^{2} \mid X_{0}=0\right]$, at time $t=1$, of the affine process on $[0, \infty)$ reflected at 0 . The row with $n=0$ gives the steady-state values. The row with $n=1$ gives the values computed by truncating the eigenfunction expansion after the term with $n=1$, and similarly for the other rows. The affine process parameters are $\sigma=\kappa=\theta=1$ and $\ell=-1$, starting at the origin.

\begin{tabular}{ccc}
\hline$n$ & $\mathrm{E}\left[X_{1} \mid X_{0}=0\right]$ & $\mathrm{E}\left[X_{1}^{2} \mid X_{0}=0\right]$ \\
\hline 0 & 1.21053 & 2.31579 \\
1 & 0.96237 & 1.37075 \\
2 & 0.95354 & 1.43163 \\
3 & 0.95231 & 1.43388 \\
4 & 0.95208 & 1.43412 \\
5 & 0.95203 & 1.43415 \\
6 & 0.95202 & 1.43416 \\
7 & 0.95202 & 1.43416 \\
\hline
\end{tabular}

\section{References}

Abate, J. And Whitt, W. (1987a). Transient behavior of regulated Brownian motion. I. Starting at the origin. Adv. Appl. Prob. 19, 560-598.

Abate, J. AND Whitt, W. (1987b). Transient behavior of regulated Brownian motion. II. Nonzero initial conditions. Adv. Appl. Prob. 19, 599-631.

Abramowitz, M. and Stegun, I. A. (1972). Handbook of Mathematical Functions. Dover, New York.

BALl, C. A. AND Roma, A. (1998). Detecting mean reversion within reflecting barriers: applications to the European exchange rate mechanism. Appl. Math. Finance 5, 1-15.

Bertolla, G. and Caballero, R. J. (1992). Target zones and realignments. Amer. Econom. Rev. 82, 520-536.

Borodin, A. N. And Salminen, P. (1996). Handbook of Brownian Motion. Birkhäuser, Boston, MA.

Buchiolz, H. (1969). The Confluent Hypergeometric Function with Special Emphasis on Its Applications (Springer Tracts Natural Philos. 15). Springer, New York.

Coffman, E. G., Puhalskit, A. A. and Reiman, M. I. (1998). Polling systems in heavy traffic: a Bessel process limit. Math. Operat. Res. 23, 257-304.

Cox, J. C., Ingersoll, J. E. ANd Ross, S. A. (1985). A theory of the term structure of interest rates. Econometrica 53, 385-407.

Davydov, D. And Linetsky, V. (2003). Pricing options on scalar diffusions: an eigenfunction expansion approach. Operat. Res. 51, 185-209.

DE Jong, F. (1994). A univariate analysis of European monetary system exchange rates using a target zone model. $J$. Appl. Econometrics 9, 31-45.

ERdelyi, A. (1953). Higher Transcendental Functions, Vol. 2. McGraw-Hill, New York.

FARnsworth, H. AND BASs, R. (2003). The term structure with semi-credible targeting. J. Finance 58, 839-865. 
Feller, W. (1951). Two singular diffusion problems. Ann. Math. 54, 173-182.

Fulton, C. ANd Pruess, S. (1994). Eigenvalue and eigenfunction asymptotics for regular Sturm-Liouville problems. J. Math. Anal. Appl. 188, 297-340.

GöInG-JAESchKe, A. AND YoR, M. (2003). A survey and some generalizations of Bessel processes. Bernoulli 9, 313-349.

Goldstein, R. And Keirstead, W. P. (1997). On the term structure of interest rates in the presence of reflecting and absorbing boundaries. Res. Rep. 97-1, Ohio State University.

Gorovor, V. AND Linetsky, V. (2004). Black's model of interest rates as options, eigenfunction expansions and Japanese interest rates. Math. Finance 14, 49-78.

Hanson, S. D., Myers, R. J. ANd Hilker, J. H. (1999). Hedging with futures and options under a truncated cash price distribution. J. Agricultural Appl. Econom. 31, 449-459.

Harrison, M. (1985). Brownian Motion and Stochastic Flow Systems. John Wiley, New York.

Itô, K. And McKean, H. (1974). Diffusion Processes and their Sample Paths. Springer, Berlin.

KARLIN, S. AND McGregor, J. (1957). The differential equations of birth-and-death processes and the Stieltjes moment problem. Trans. Amer. Math. Soc. 85, 489-546.

Karlin, S. and TaYlor, H. M. (1981). A Second Course in Stochastic Processes. Academic Press, San Diego, CA.

Krugman, P. R. (1991). Target zones and exchange rate dynamics. Quart. J. Econom. 106, 669-682.

LANGER, H. AND SCHENK, W. S. (1990). Generalized second-order differential operators, corresponding gap diffusions and superharmonic transformations. Math. Nachr. 148, 7-45.

Lebedev, N. N. (1972). Special Functions and Their Applications. Dover, New York.

LEVINSON, N. (1951). A simplified proof of the expansion theorem for singular second order differential operators. Duke Math. J. 18, 57-71.

Levitan, B. M. (1950). Expansion in Characteristic Functions of Differential Equations of the Second Order. Gostekhizdat, Moscow (in Russian).

Levitan, B. M. And Sargsjan, I. S. (1975). Introduction to Spectral Theory. American Mathematical Society, Providence, RI.

LEWIS, A. (1998). Applications of eigenfunction expansions in continuous-time finance. Math. Finance 8, 349-383.

Linetsky, V. (2004a). Computing hitting times of OU and affine diffusions: applications to mean-reverting models. $J$. Comput. Finance 7, 1-22.

LinETSKY, V. (2004b). Lookback options and diffusion hitting times: a spectral expansion approach. Finance Stoch. 8, 373-398.

LINETSKY, V. (2004c). Spectral expansions for Asian (average price) options. Operat. Res. 52, 856-867.

Linetsky, V. (2004d). The spectral decomposition of the option value. Internat. J. Theoret. Appl. Finance 7, 337-384.

LinetsKy, V. (2004e). The spectral representation of Bessel processes with drift: applications in queueing and finance. J. Appl. Prob. 41, 327-344.

McKean, H. (1956). Elementary solutions for certain parabolic partial differential equations. Trans. Amer. Math. Soc. 82, 519-548.

Pitman J. W. And Yor, M. (1982). A decomposition of Bessel bridges. Z. Wahrscheinlichkeitsth. 59, $425-457$.

RicCIARDI, L. M. AND SACERDOTE, L. (1987). On the probability densities of an Ornstein-Uhlenbeck process with a reflecting boundary. J. Appl. Prob. 24, 355-369.

Schobel, R. AND ZHU, J. (1999). Stochastic volatility with an Ornstein-Uhlenbeck process: an extension. Europ. Finance Rev. 3, 23-46.

Shiga, T. And Watanabe, S. (1973). Bessel diffusions as a one-parameter family of diffusion processes. Z. Wahrscheinlichkeitsth. 27, 37-46.

SRIKant, R. And Whitt, W. (1996). Simulation run lengths to estimate blocking probabilities. ACM Trans. Model. Comput. Simul. 6, 7-52.

Slater, L. J. (1960). Confluent Hypergeometric Functions. Cambridge University Press.

Svensson, L. E. O. (1991). The term structure of interest rate differentials in a target zone. Theory and Swedish data. J. Monetary Econom. 28, 87-116.

Veestraeten, D. (2004). The conditional probability density function for a reflected Brownian motion. Comput. Econom. 23, 185-207.

Ward, A. R. AND GlynN, P. W. (2003a). A diffusion approximation for a Markovian queue with reneging. Queueing Systems 43, 103-128.

Ward, A. R. ANd GLYnN, P. W. (2003b). Properties of the reflected Ornstein-Uhlenbeck process. Queueing Systems 44, 109-123.

Whitt, W. (2004). A diffusion approximation for the G/GI/n/m queue. Operat. Res. 52, 922-941.

Whit, W. (2005). Heavy-traffic limits for the $\mathrm{G} / \mathrm{H} 2 * / n / m$ queue. Math. Operat. Res. 30, 1-27.

Wong, E. (1964). The construction of a class of stationary Markoff processes. In Proc. Symp. Appl. Math. Vol. XVI, ed. R. Bellman, American Mathematical Society, Providence, RI, pp. 264-276. 